\title{
Effects, barriers and facilitators in predischarge home assessments to improve the transition of care from the inpatient care to home in adult patients: An integrative review
}

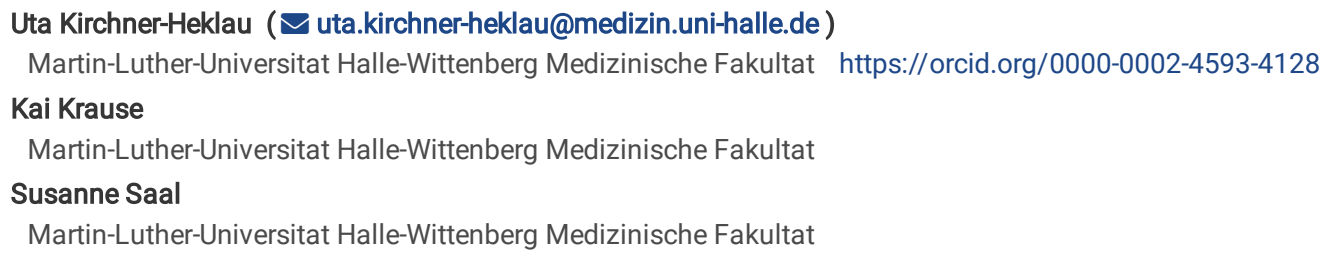

\section{Research article}

Keywords: Patient discharge, home visit, systematic review, occupational therapy, rehabilitation, virtual reality

Posted Date: December 14th, 2020

DOI: https://doi.org/10.21203/rs.3.rs-28272/v2

License: @ (i) This work is licensed under a Creative Commons Attribution 4.0 International License. Read Full License

Version of Record: A version of this preprint was published at BMC Health Services Research on June 2nd, 2021. See the published version at https://doi.org/10.1186/s12913-021-06386-4. 


\section{Abstract}

Background: Predischarge home assessments (PDHA) aim to support safe discharge from hospital or rehabilitation. There is insufficient evidence on the effectiveness of PDHA. For adults with any diagnosis, we aimed to determine (1) the effects of PDHA on outcomes associated with the successful return to community living (e.g., Activities of Daily Living, falls) and (2) the associated barriers and facilitators in order to derive recommendations for clinical practice.

Methods: We searched Medline, EMBASE, CINAHL, five additional databases and other sources. We included individual and cluster randomized (RCT/CRCT) and controlled clinical trials comparing PDHA versus usual care/other intervention, as well as qualitative/mixed methods studies dealing with PDHA. Critical appraisal was performed according to the Cochrane risk-of-bias tool in quantitative studies and the Critical Appraisal Skills Programme (CASP) as well as the McMaster University Guidelines for Critical Review Form for qualitative studies and data extraction. Meta-analysis, thematic synthesis and integrative synthesis were performed.

Results: EightRCTs ( $n=1149)$ and eleven qualitative studies $(n=346)$ met the inclusion criteria. RCTs reported a variety of outcomes ( $n=17)$. We are uncertain if PDHA has any effect on patient outcomes in Activities of Daily Living, quality of life, mobility and fear of falling, falls and hospital readmissions (with moderate to very low quality of the evidence). The qualitative studies revealed facilitators and barriers which should be considered by therapists when conducting PDHA. These were related to the following topics: patient safety education, patient information, patients' acceptance of modifications and aids, functional assessment, standardization of procedures as well as the consideration of relevant patient conditions and contextual factors in PDHA.

Conclusion: There is no evidence from the meta-analysis for the effectiveness of PDHA. Further robust studies are needed to adapt and evaluate PDHA interventions, taking the identified stakeholders' views on PDHA into account and following the current recommendations for the development and evaluation of complex interventions.

\section{Trial registration:}

The review was registered and methods were reported on PROSPERO on 18 ${ }^{\text {th }}$ July 2018 (https://www.crd.york.ac.uk/PROSPERO. CRD42018100636).

\section{Background}

Discharge planning aims to support patients' independence in activities of daily living (ADL) and participation in life and to support a safe home environment to prevent falls and injuries that could lead to hospital readmissions; therefore, predischarge home assessments are an important component of discharge planning.

Predischarge home assessments (PDHA) are conducted while the patients are in hospital or inpatient rehabilitation in order to gain information for therapy and discharge management including the provision of aids and home modifications before the transition to the patient's home (or nursing home)[9]. The way in which information about the home environment is gathered varies [3-5]. Physical home visits with the patient are described as costly and time consuming $[3,6,7]$. The ward-based collection of environmental information data can be obtained by interviews. These interviews can be supported by the technological visualization of the home environment[2-4]. Access visits are made to gain information about the patient's home without the patient being present [4, 8]. Therefore, access visits and all types of ward-based assessments do separately and independently assess both the home environment and the patient's functioning. An assessment of the patient's functioning within his/her specific home environment can only be provided during occupational therapy visits when the patient is present. All types of home assessments by occupational therapists aim at preparing and improving the patient's hospital discharge to his previous or a new residence, respectively and are considered in this study under the term PDHA.

There is limited evidence on the effects of PDHA. A recent systematic review analyzing the effects of predischarge home visits and their influencing factors [5] included five RCTs, one cohort study and three retrospective medical record/chart audits as well as four interview studies and one questionnaire survey. The studies were of low to moderate quality and reported a small decrease in the risk of falling, but no other statistically significant effects.

In recent years, studies on new technologies for PDHA have been published, which were not included in the review by Lockwood et al. [5]. These studies focus on $3 \mathrm{D}$ visualization that offers computer-generated environments, scenarios and objects $[3,4,10]$ that can be used to avoid travelling to a patient's home and to improve the patient's involvement in home modification planning. Thus, an update of the evidence synthesis on PDHA is warranted.

There is some information about stakeholders' views on the PDHA process. In their review, Lockwood et al. also investigated the patients' and carers' perceptions of PDHA effectiveness and included five qualitative studies, reporting on three emerging themes: satisfaction with the process, purpose of the visit, and incorporation of patient and carer opinions in the decision-making process [5]. The authors concluded that it might have an impact on the effects of the intervention and how PDHA are conducted, and recommended consultation and patients' participation in the PDHA process [5]. A thematic synthesis included five qualitative studies and reported the experiences and perceptions of older adults concerning PDHA. It is required that patients understand the purpose of PDHA and therapists are open-minded towards the coping strategies of older adults [11]. In recent years, a number of qualitative studies investigating the views of stakeholders of the PDHA process have been published.

Therefore, we conducted a mixed methods review aiming to determine the effects of PDHA on outcomes associated with a successful return to community living and to update the evidence on barriers and facilitators in the PDHA process to derive recommendations for improving PDHA.

\section{Methods}


The review was registered in the International Prospective Register of Systematic Reviews (PROSPERO) under the identification number CRD42018100636. The protocol and the review were reported according the recommendations of the Preferred Reporting Items for Systematic Reviews and Meta-Analyses statement (PRISMA) [12] and the framework to enhance transparency in reporting the synthesis of qualitative research (ENTREQ) [13].

\section{Eligibility criteria}

Quantitative clinical trials were included if

1. an individual or cluster-randomized controlled or controlled trial design was used,

2. the study participants were aged $\geq 18$ years, admitted to hospitals or rehabilitation facilities with any diagnosis at all. Studies with participants in psychiatric and perinatologic settings were excluded.

3. PDHA was reported as a primary intervention, though it could vary in purpose (e.g., discharge planning or functional assessment), delivery mode (e.g., with / without a patient), intensity, length and frequency,

4. PDHA was compared to another intervention or to usual care,

5. the outcomes were associated with a successful return to community living (e.g., functioning in the home environment, readmissions), quality of life, patient satisfaction, caregiver burden and / or the immediate output of the predischarge home assessment (e.g., home modifications), and if

6. the study was published in the English or German language.

Qualitative studies and mixed methods studies were included if they reported on views and opinions, perspectives, beliefs, feelings, understanding, experiences or behavior regarding PDHA of adult stakeholders (e.g., patients, healthcare providers) and were published in the English or German language.

\section{Search strategy and selection criteria}

In July 2018, Medline, EMBASE, CINAHL, the Cochrane Central Register of Controlled Trials (CENTRAL) PEDro and OTseeker databases and trial registries (PROSPERO and ICTRP) were searched. The overall search strategy used the following combined search terms for database searches: discharge, inpatient, sub-acute care, acute care, rehabilitation [MeSH], House Calls [Mesh], home visiting, home visit, environmental assessment, assessment visit, home safety, home modification, environmental modification, weekend passes, weekend pass (see Additional File 1 for search strategy). Relevant RCTs, CCTs and qualitative studies were included in the analysis. References of the identified publications were checked from July 2018 until December 2018 . A literature update was undertaken between the $9^{\text {th }}$ and $14^{\text {th }}$ of September 2020. Forward citation search was conducted using Google Scholar and Web of Science.

\section{Data collection and analysis}

\section{Study selection}

Two independent reviewers $(\mathrm{KK}, \mathrm{UKH})$ applied the inclusion and exclusion criteria to titles and abstracts of the search results. Discrepancies were discussed and resolved by consensus with a third author (SuS) and by reading the full text, if needed. The remaining sample of studies was read in full text by two independent reviewers (KK, UKH). Discrepancies were discussed and resolved by consensus with a third author (SuS). Inclusion was unclear in one case (Gursen, 2003) due to insufficient reporting on the study design. After trying to contact the authors without any success, we excluded the study. Multiple publications reporting on the same study were clustered and handled as one unit. See Additional File 1 for excluded studies.

\section{Data extraction and management}

One reviewer extracted the descriptive information from the publications using a piloted data extraction sheet, and another reviewer double-checked the extracted data. The following information was extracted for quantitative and qualitative studies: aim and focus of the studies, study design, details about the intervention according to the TiDier Checklist [14], number and characteristics of participants, outcomes, and outcome measures. For quantitative studies, raw scores were extracted using Excel sheets. If outcomes were measured at multiple time points, the latest follow-up was selected. If studies reported on outcomes using more than one measure, we used only one measure per outcome, according to a pre-specified hierarchy, determined by the researcher group (see Additional File 2). For qualitative studies, we extracted verbatim quotes from study participants and the authors' descriptions of the findings from the results section.

\section{Risk of bias assessment}

Two reviewers (KK, UKH) independently assessed the risk of bias. Any disagreements were resolved by discussion and, if necessary, by consulting a third author (SuS). We used the methods and recommendations for the assessment of risk of bias and heterogeneity in individual quantitative studies as described in the Cochrane Handbook 5.1.0 [16].

For qualitative studies, a set of criteria from the CASP tool [17] as well as from the Guidelines for Critical Review Form: Qualitative Studies, Version 2 [18] was used to assess the internal validity (see Additional File 3 for quality appraisal of qualitative studies). 


\section{Data analysis and synthesis}

In case only median, sample size and interquartile range (with first and third interquartile) were presented and imputing SDs was not possible, we estimated the sample mean and standard deviation according to Wan et al. [19]. Meta-analysis was conducted using a random-effects model (REM). We decided to use and report the effects in the fixed effect model additionally, if the heterogeneity was rather low, since the random effects model makes some assumptions of its own about the distribution of the study effects, which may not be accurate due to the small number of studies. [16]. For continuous outcome data, we used standardized mean differences (SMD) with 95\% confidence intervals (Cls) for different scales or units and mean differences (MD) with standard deviations (SD) for same scales. For meta-analysis in dichotomous data, we calculated risk ratios (RR), which were defined as the average number of events per participant, with $95 \% \mathrm{Cls}$. We used the $\mathrm{I}^{2}$ test for the assessment of statistical_heterogeneity, a significance level of $p$ less than 0.10 , and the chi-squared test.

We assumed that effect sizes may differ due to different scales per outcome and conducted a corresponding sensitivity analysis. Due to the small number of studies that differed per pooled outcome in several aspects (diagnosis, type of intervention, time of measurement) at the same time, we decided to forgo a post-hoc subgroup analysis, according to the recommendations of the Cochrane Handbook for Systematic Reviews of Interventions Version 5 [20]. To judge the quality of the evidence, the GRADE approach [21] was used. See Additional File 5 for details on our GRADE ratings.

Qualitative data were entered verbatim using MAXQDA 2018.2 software for data analysis. Thematic analysis was applied [22]. Line-by-line coding was performed by two reviewers independently to reconcile comprehension. The descriptive themes were discussed iteratively with the whole team (KK, UKH, and SuSa) until a consensus regarding comprehensibility and distinction of themes was reached. We synthesized findings according to the emerging themes related to barriers and facilitators of the PDHA process. Implications for practice and intervention development were inferred, according to the methods for thematic synthesis by Thomas, [15]. Based on the facilitating factors and barriers in certain subject areas, we elaborated how a PDHA intervention should be designed in order to take into account the views of patients and healthcare professionals.

An integrative synthesis of quantitative results and implications from qualitative studies was performed, whereby two reviewers (KK, UKH) examined the intervention descriptions of the included RCTs to identify whether implications were addressed or not. The detailed information supporting the decisions was discussed and documented. A matrix of the integrative synthesis mapped the studies' effect sizes with contextual details and information on corresponding implications and interventions (please see Additional File 8).

\section{Results}

\section{Study selection}

Our search revealed 3271 publications (Figure 1), of which 23 publications met our inclusion criteria. These publications referred to eight RCTs and 11 qualitative studies.

\section{Study characteristics}

Eight RCTs with 1072 participants were included [2, 4, 8, 23-30]. The size of the studies ranged from ten to 400 participants. The cohort study alongside one RCT was not considered in the analysis [8].

Eleven qualitative studies with a total of 346 participants (range: $n=4$ [36] to $n=122$ [40]) were included. All the studies used interview techniques, one used participant observation additionally [37], and another one used a semistructured survey [40]. Three studies explored perceptions of patients, therapists and older adults in community dwellings with regard to the use of virtual reality (VR) applications in PDHA [10, 33, 34]. Three studies explored factors considered by OTs when deciding about stroke patients' need for a predischarge home assessment visit, as well as clinical reasoning and practice of PDHA [34, 38-40]. One study focused on older adults' and carers' perception of and involvement in PDHA decision-making processes [32]. Another study also highlighted the patients' perspective on PDHA [36]. A summary of characteristics of the included quantitative and qualitative studies is displayed in Table 1.

Table 1: Study characteristics of included studies 


\begin{tabular}{|c|c|c|c|c|c|c|}
\hline Reference & $\begin{array}{l}\text { Study } \\
\text { design, } \\
\text { country, } \\
\text { setting }\end{array}$ & $\begin{array}{l}\text { Participants } \\
\text { Number, } \\
\text { age in years, } \\
\text { percent female } \\
(\square \%)\end{array}$ & $\begin{array}{l}\text { Intervention } \\
\text { description }\end{array}$ & Outcomes/aim of qualitative research & Outcome measures & $\begin{array}{l}\text { FU } \\
\text { in } \\
\text { mo } \\
\text { nths }\end{array}$ \\
\hline $\begin{array}{l}\text { Clemson et } \\
\text { al., } 2016 \\
\text { [2] }\end{array}$ & $\begin{array}{l}\text { RCT } \\
\text { Australia } \\
\text { Acute care, } \\
\text { unspecified }\end{array}$ & $\begin{array}{l}\mathrm{n}=400 \\
\text { Intervention } 80.2 \\
( \pm 6.4 \text {, range n.r.) } \\
\text { Control } \\
\text { Control } \\
80.7 \text { ( } \pm 5.7 \text {, } \\
\text { range n.r.) } \\
\square 63.9 \%\end{array}$ & $\begin{array}{l}\text { Intervention } \\
\text { In-hospital rapport } \\
\text { building, } \\
\text { interview, ADL- } \\
\text { assessment, } \\
\text { predischarge home } \\
\text { visit, post- } \\
\text { discharge home- } \\
\text { visit, telephone } \\
\text { calls } \\
\text { Control } \\
\text { Usual care, in- } \\
\text { hospital interview, } \\
\text { ADL assessment, } \\
\text { access visit if } \\
\text { required }\end{array}$ & $\begin{array}{l}\text { Functional independence, } \\
\text { participation in ADL, unplanned readmissions, } \\
\text { emergency department visits, recommendations }\end{array}$ & $\begin{array}{l}\text { NEADL [41], Late Life } \\
\text { Disability Index (LLDI) - sub } \\
\text { scores: frequency and } \\
\text { limitation [60], } \\
\text { number of: recommendations, } \\
\text { unplanned readmissions, } \\
\text { emergency department visits, } \\
\text { falls, process outcomes (e.g., } \\
\text { number of prescribed and } \\
\text { tried equipment; effects not } \\
\text { estimated) }\end{array}$ & 3 \\
\hline $\begin{array}{l}\text { Drummond } \\
\text { et al., } 2013 \\
\text { [8] }\end{array}$ & $\begin{array}{l}\text { RCT } \\
\text { UK } \\
\text { Stroke } \\
\text { rehabilitation unit }\end{array}$ & $\begin{array}{l}\mathrm{n}=126 \\
\text { Intervention } \\
70.64( \pm 14.29, \\
\text { range } 34-88) \\
\square 54.7 \% \\
\text { Control } \\
73.65( \pm 16.06, \\
\text { range } 41-99) \\
\square 47.8 \%\end{array}$ & $\begin{array}{l}\text { Intervention } \\
\text { One or two } \\
\text { predischarge home } \\
\text { visits } \\
\text { Control } \\
\text { Structured home } \\
\text { assessment } \\
\text { interview }\end{array}$ & $\begin{array}{l}\text { ADL/IADL, mobility, unplanned readmissions, falls, } \\
\text { emotional distress in medical settings, depressed mood } \\
\text { of clients with stroke and significant aphasia, caregiver } \\
\text { strain }\end{array}$ & $\begin{array}{l}\text { NEADL [41], Barthel Index } \\
\text { [61], RMI [46], Number of } \\
\text { unplanned readmissions, GHQ- } \\
28 \text { [49], SADQ-10 [51], } \\
\text { Caregiver Strain Index [52] }\end{array}$ & 1 \\
\hline $\begin{array}{l}\text { Hagsten et } \\
\text { al., } 2004 \\
{[23]} \\
\text { Hagsten et } \\
\text { al., } 2006 \\
{[24]}\end{array}$ & $\begin{array}{l}\text { RCT } \\
\text { Sweden } \\
\text { Acute care, hip } \\
\text { fractures }\end{array}$ & $\begin{array}{l}\mathrm{n}=100 \\
\text { Intervention } \\
81( \pm 23, \text { range } \\
68-91) \\
84 \% \\
\text { Control } \\
79( \pm 30 \text {, range } \\
65-95) \\
76 \%\end{array}$ & $\begin{array}{l}\text { Intervention } \\
\text { Individual daily } \\
\text { training, including } \\
\text { use of technical } \\
\text { aids, single } \\
\text { predischarge home } \\
\text { visit } \\
\text { Control } \\
\text { One session of } \\
\text { walking } \\
\text { instruction when } \\
\text { in hospital }\end{array}$ & ADL/IADL, health-related quality of life & $\begin{array}{l}\text { ADL [62], EQ5D [63], IADL } \\
\text { single scales: moving around } \\
\text { indoors; performance of light } \\
\text { housework; getting in and out } \\
\text { of a car, SWED-QUAL [43] } \\
\text { subscales }\end{array}$ & 2 \\
\hline $\begin{array}{l}\text { Lannin et } \\
\text { al., } 2007 \\
{[25]}\end{array}$ & $\begin{array}{l}\text { RCT } \\
\text { Australia } \\
\text { Rehabilitation } \\
\text { unit, mixed } \\
\text { (cardiac, } \\
\text { orthopedic trauma, } \\
\text { neurological, } \\
\text { orthopedic joint } \\
\text { surgery, spinal or } \\
\text { deconditioned) }\end{array}$ & $\begin{array}{l}\mathrm{n}=10 \\
\text { Intervention } \\
80.0 \text { ( } \pm 7 \text {, range } \\
\text { n.r.) } \\
\square 100 \% \\
\text { Control } \\
82.4 \text { ( } \pm 7 \text {, range } \\
\text { n.r.) } \\
\square 60 \%\end{array}$ & $\begin{array}{l}\text { Intervention } \\
\text { Single } \\
\text { predischarge home } \\
\text { visit } \\
\text { Control } \\
\text { In-hospital } \\
\text { consultation prior } \\
\text { to discharge }\end{array}$ & $\begin{array}{l}\text { ADL/IADL, mobility, unplanned readmissions, falls, fear } \\
\text { of falling, community support, health-related quality of } \\
\text { life }\end{array}$ & $\begin{array}{l}\text { NEADL [41], FIM [64], RNLI - } \\
\text { Reintegration to Normal } \\
\text { Living Index [65], Tinnetti } \\
\text { [45], number of unplanned } \\
\text { readmissions, number of falls, } \\
\text { FES-I [47], EQ5D [63], EQ-5D } \\
\text { VAS [44] }\end{array}$ & 7 \\
\hline $\begin{array}{l}\text { Lockwood } \\
\text { et al., } 2019 \\
{[26]}\end{array}$ & $\begin{array}{l}\text { RCT } \\
\text { Australia } \\
\text { Acute and } \\
\text { rehabilitation } \\
\text { (cardiac, } \\
\text { orthopaedic } \\
\text { trauma, } \\
\text { neurological, } \\
\text { orthopaedic joint } \\
\text { surgery, spinal or } \\
\text { deconditioned) }\end{array}$ & $\begin{array}{l}\mathrm{n}=77 \\
\text { Intervention } \\
83.4 \text { ( } \pm 7.1 \text {, } \\
\text { range n.r.) } \\
\square 76 \% \\
\text { Control } \\
80.9 \text { ( } \pm 7.3 \text {, } \\
\text { range n.r.) } \\
\square 68 \%\end{array}$ & $\begin{array}{l}\text { Intervention } \\
\text { Single } \\
\text { predischarge home } \\
\text { visit and usual } \\
\text { care } \\
\text { Control } \\
\text { usual care }\end{array}$ & $\begin{array}{l}\text { ADL/IADL, health-related quality of life, unplanned } \\
\text { readmissions, number of days in hospital after index } \\
\text { discharge, falls, fear of falling, adverse events }\end{array}$ & $\begin{array}{l}\text { NEADL [41], SMAF [42], FIM } \\
\text { [66], FES-I [47], EQ5D[63], } \\
\text { EQ-5D VAS [44] }\end{array}$ & 6 \\
\hline $\begin{array}{l}\text { Lockwood } \\
\text { et al., } 2020 \\
{[27] £}\end{array}$ & $\begin{array}{l}\text { RCT, process } \\
\text { evaluation } \\
\text { Australia } \\
\text { Acute and } \\
\text { rehabilitation } \\
\text { (cardiac, } \\
\text { orthopaedic }\end{array}$ & $\begin{array}{l}\mathrm{n}=77 \\
\text { Intervention } \\
83.4 \text { ( } \pm 7.1, \\
\text { range n.r.) } \\
\square 76 \% \\
\text { Control } \\
80.9 \text { ( } \pm 7.3 \text {, } \\
\text { range n.r.) } \\
\square 68 \%\end{array}$ & $\begin{array}{l}\text { Intervention } \\
\text { Single } \\
\text { predischarge home } \\
\text { visit and usual } \\
\text { care } \\
\text { Control } \\
\text { usual care }\end{array}$ & Recommendations, adherence to recommendations & $\begin{array}{l}\text { Number of recommendations, } \\
\text { Number of adherence to } \\
\text { recommendations }\end{array}$ & 1 \\
\hline
\end{tabular}




\begin{tabular}{|c|c|c|c|c|c|c|}
\hline & $\begin{array}{l}\text { trauma, } \\
\text { neurological, } \\
\text { orthopaedic joint } \\
\text { surgery, spinal or } \\
\text { deconditioned) }\end{array}$ & & & & & \\
\hline $\begin{array}{l}\text { Nikolaus et } \\
\text { al., } 2003 \\
\text { [9] }\end{array}$ & $\begin{array}{l}\text { RCT } \\
\text { Germany } \\
\text { Geriatric acute } \\
\text { care, unspecified }\end{array}$ & $\begin{array}{l}\mathrm{n}=360 \\
\text { Intervention } \\
81.2 \\
( \pm 6.2, \text { range } \\
84.9-87.5) \\
\square 72.4 \% \\
\text { Control } \\
81.9 \\
( \pm 6.5, \text { range } \\
74.4-88.4) \\
\square 74.3 \%\end{array}$ & $\begin{array}{l}\text { Intervention } \\
\text { Predischarge home } \\
\text { visit and post- } \\
\text { discharge follow- } \\
\text { up visit(s), } \\
\text { comprehensive in- } \\
\text { hospital geriatric } \\
\text { assessment } \\
\text { Control } \\
\text { Comprehensive } \\
\text { geriatric } \\
\text { assessment and } \\
\text { usual care }\end{array}$ & Falls, recommendations & $\begin{array}{l}\text { Number of falls, compliance } \\
\text { with recommendations after } 12 \\
\text { months }\end{array}$ & 12 \\
\hline $\begin{array}{l}\text { Pardessus } \\
\text { et al., } 2002 \\
{[28]}\end{array}$ & $\begin{array}{l}\text { RCT } \\
\text { France } \\
\text { Geriatric acute } \\
\text { care, unspecified }\end{array}$ & $\begin{array}{l}\mathrm{n}=60 \\
\text { Intervention } \\
83.51 \text { ( } \pm 9.08, \\
\text { range n.r.) } \\
\text { प } 76 \% \\
\text { Control } \\
82.9 \text { ( } \pm 6.33, \\
\text { range n.r.) } \\
\square 80 \%\end{array}$ & $\begin{array}{l}\text { Intervention } \\
\text { Predischarge home } \\
\text { visit } \\
\text { Control } \\
\text { Usual care }\end{array}$ & $\begin{array}{l}\text { ADL/IADL, } \\
\text { Falls, re-hospitalization, institutionalization }\end{array}$ & $\begin{array}{l}\text { IADL [67], SMAF subscales } \\
\text { [42] ADL subscales [68] } \\
\text { number of recurring falls, } \\
\text { mean number of fall } \\
\text { recurrence in former fallers, } \\
\text { number of re-hospitalizations, } \\
\text { number of institutionalizations }\end{array}$ & 12 \\
\hline $\begin{array}{l}\text { Provencher } \\
\text { et al., } 2020 \\
{[29]^{\S}}\end{array}$ & $\begin{array}{l}\text { RCT, Post-hoc } \\
\text { analysis } \\
\text { Australia } \\
\text { Acute care, } \\
\text { unspecified }\end{array}$ & $\begin{array}{l}\mathrm{n}=400 \\
\text { Intervention } 80.2 \\
( \pm 6.4 \text {, range n.r.) } \\
\square 59.6 \% \\
\text { Control } \\
80.7 \text { ( } \pm 5.7 \text {, } \\
\text { range n.r.) } \\
\square 63.9 \%\end{array}$ & $\begin{array}{l}\text { Intervention } \\
\text { In-hospital rapport } \\
\text { building, } \\
\text { interview, ADL- } \\
\text { assessment, } \\
\text { predischarge home } \\
\text { visit, post- } \\
\text { discharge home } \\
\text { visit, telephone } \\
\text { calls } \\
\text { Control } \\
\text { Usual care, in- } \\
\text { hospital interview, } \\
\text { ADL assessment, } \\
\text { access visit if } \\
\text { required }\end{array}$ & $\begin{array}{l}\text { Functional independence, } \\
\text { participation in ADL, unplanned readmissions, } \\
\text { emergency department visits, recommendations }\end{array}$ & $\begin{array}{l}\text { NEADL [34], Late Life } \\
\text { Disability Index (LLDI) - sub } \\
\text { scores: frequency and } \\
\text { limitation [52], } \\
\text { number of: recommendations, } \\
\text { unplanned readmissions, } \\
\text { emergency department visits, } \\
\text { falls, process outcomes (e.g., } \\
\text { number of prescribed and } \\
\text { tried equipment; effects not } \\
\text { estimated) }\end{array}$ & 3 \\
\hline $\begin{array}{l}\text { Threapleton } \\
\text { et al., } 2018 \\
{[4]}\end{array}$ & $\begin{array}{l}\text { RCT } \\
\text { UK } \\
\text { Stroke ward, acute } \\
\text { care }\end{array}$ & $\begin{array}{l}\mathrm{n}=16 \\
\text { Intervention } \\
72( \pm 21.08, \\
\text { range } 38-90) \\
\square 75 \% \\
\text { Control } \\
70( \pm 12.6, \\
\text { range } 46-86) \\
\square 3 \%\end{array}$ & $\begin{array}{l}\text { Intervention } \\
\text { Single } \\
\text { predischarge } \\
\text { virtual home } \\
\text { assessment } \\
\text { Control } \\
\text { Usual care }\end{array}$ & $\begin{array}{l}\text { ADL/IADL, } \\
\text { overall independence, } \\
\text { mobility, } \\
\text { fear of falling, } \\
\text { health-related quality of life }\end{array}$ & $\begin{array}{l}\text { NEADL [41], Barthel- Index } \\
\text { [61], MRS [69], Rivermead } \\
\text { Mobility Index [46], FES-I } \\
\text { [47], EQ5D [63] }\end{array}$ & 6 \\
\hline $\begin{array}{l}\text { Wales et al., } \\
2018[30]^{\S}\end{array}$ & $\begin{array}{l}\begin{array}{l}\text { RCT, economic } \\
\text { evaluation }\end{array} \\
\text { Australia } \\
\text { Acute care, } \\
\text { unspecified }\end{array}$ & $\begin{array}{l}\mathrm{n}=400 \\
\text { Intervention } 80.2 \\
( \pm 6.4 \text {, range n.r.) } \\
\square 59.6 \% \\
\text { Control } \\
80.7 \text { ( } \pm 5.7 \text {, } \\
\text { range n.r.) } \\
\square 63.9 \%\end{array}$ & $\begin{array}{l}\text { Intervention } \\
\text { In-hospital rapport } \\
\text { building, } \\
\text { interview, ADL- } \\
\text { assessment, } \\
\text { predischarge home } \\
\text { visit, post- } \\
\text { discharge home } \\
\text { visit, telephone } \\
\text { calls } \\
\text { Control } \\
\text { Usual care, in- } \\
\text { hospital interview, } \\
\text { ADL assessment, } \\
\text { access visit if } \\
\text { required }\end{array}$ & Costs for predischarge home visits & $\begin{array}{l}\text { costs for occupational therapy } \\
\text { time, travel, community follow } \\
\text { up, hospital readmission }\end{array}$ & 3 \\
\hline $\begin{array}{l}\text { Atwal et al., } \\
2008 \text { [32] }\end{array}$ & $\begin{array}{l}\text { Semi-structured } \\
\text { interview } \\
\text { UK } \\
\text { Geriatric acute } \\
\text { care }\end{array}$ & $\begin{array}{l}\text { Patients, main } \\
\text { carers } \\
\mathrm{n}=15 \\
86,46 \text { years } \\
\text { (range } 73-97 \text { ) } \\
\square 60 \%\end{array}$ & $\begin{array}{l}\text { Intervention } \\
\text { Single } \\
\text { predischarge home } \\
\text { visit }\end{array}$ & $\begin{array}{l}\text { - To explore older adults' and carers' involvement in } \\
\text { decisions that were made during the home visit; } \\
\text { - To explore older adults' and carers' perceptions of the } \\
\text { home visit process }\end{array}$ & n.a. & n.a. \\
\hline
\end{tabular}

Page 6/20 


\begin{tabular}{|c|c|c|c|c|c|c|}
\hline $\begin{array}{l}\text { Atwal et al., } \\
2014 \text { a [33] }\end{array}$ & $\begin{array}{l}\text { Semi-structured } \\
\text { interview; think } \\
\text { aloud technique } \\
\text { UK } \\
\text { Acute care and } \\
\text { community care }\end{array}$ & $\begin{array}{l}\text { OTs } \\
\mathrm{n}=7 \\
\square 71 \% \\
\text { social services, } \\
\text { older persons, } \\
\text { mental health, } \\
\text { acute care, } \\
\text { pediatrics }\end{array}$ & $\begin{array}{l}\text { Intervention } \\
\text { Virtual reality } \\
\text { predischarge home } \\
\text { assessment with } \\
\text { interior design } \\
\text { application }\end{array}$ & $\begin{array}{l}\text { - To explore occupational therapists' perceptions of a } \\
\text { virtual reality interior design application (VRIDA); } \\
\text { - to gain insights into the feasibility of using VRIDA as a } \\
\text { tool to aid the predischarge home visit (perceived } \\
\text { usefulness, perceived ease of use, actual use) }\end{array}$ & n.a. & n.a. \\
\hline $\begin{array}{l}\text { Atwal et al., } \\
2014 \text { b [34] }\end{array}$ & $\begin{array}{l}\text { Semi-structured } \\
\text { interview } \\
\text { UK } \\
\text { Acute care, } \\
\text { intermediate care, } \\
\text { rehabilitation, } \\
\text { older patients, } \\
\text { mental health } \\
\text { (older people) }\end{array}$ & $\begin{array}{l}\text { OTs } \\
n=21\end{array}$ & $\begin{array}{l}\text { Intervention } \\
\text { Predischarge home } \\
\text { visit / access visit }\end{array}$ & $\begin{array}{l}\text { - To explore occupational therapists' perceptions of home } \\
\text { visits; } \\
\text { - To ascertain occupational therapists' clinical reasoning } \\
\text { with respect to conducting home visits }\end{array}$ & n.a. & n.a. \\
\hline $\begin{array}{l}\text { Cameron et } \\
\text { al., } 2014 \\
{[35]}\end{array}$ & $\begin{array}{l}\text { In-depth interview, } \\
\text { semi-structured, } \\
\text { focus groups } \\
\text { Canada } \\
\text { Rehabilitation } \\
\text { facility, stroke }\end{array}$ & $\begin{array}{l}\text { Patients } \\
\mathrm{n}=16 \\
62 \text { years } \\
\text { (range } 25-87 \text { ) } \\
\square 75 \% \\
\text { Family } \\
\text { caregivers } \mathrm{n}=15 \\
41 \text { years } \\
\text { (range } 23 \text { - 75) } \\
\square 86,7 \% \\
\text { Multiple health } \\
\text { professionals } \\
\mathrm{n}=20\end{array}$ & $\begin{array}{l}\text { Intervention } \\
\text { Single } \\
\text { predischarge home } \\
\text { visit or } \\
\text { preparation in } \\
\text { hospital and } \\
\text { single/multiple } \\
\text { predischarge } \\
\text { weekend passes }\end{array}$ & $\begin{array}{l}\text { - To explore stroke survivors', caregivers', and } \\
\text { healthcare professionals' perceptions of weekend passes } \\
\text { offered during inpatient rehabilitation and its role in } \\
\text { facilitating the transition home }\end{array}$ & n.a. & n.a. \\
\hline $\begin{array}{l}\text { Davis et al., } \\
2019 \text { [40] }\end{array}$ & $\begin{array}{l}\text { Semi-structured } \\
\text { survey } \\
\text { Republic of Ireland } \\
\text { acute settings, } \\
\text { rehabilitation } \\
\text { settings and } \\
\text { convalescence } \\
\text { settings, adult } \\
\text { patients (over } 18 \\
\text { years) }\end{array}$ & $\begin{array}{l}\text { OTs } \\
n=122\end{array}$ & $\begin{array}{l}\text { Intervention } \\
\text { Pre-discharge } \\
\text { home visit }\end{array}$ & $\begin{array}{l}\text { To investigate clinical practice during DPHV and the } \\
\text { clinical reasoning guiding occupational therapists within } \\
\text { an Irish context }\end{array}$ & $\begin{array}{l}\text { n.a. for semistructured part } \\
\text { quantitative part of survey: } \\
\text { use of standardized tool, } \\
\text { contents of home visit bag, } \\
\text { numbers of recommendations, } \\
\text { consensus on clinical practice }\end{array}$ & n.a. \\
\hline $\begin{array}{l}\text { Godfrey et } \\
\text { al., } 2019 \\
{[39]}\end{array}$ & $\begin{array}{l}\text { Focus-group } \\
\text { interviews } \\
\text { Australia } \\
\text { Acute or sub-acute } \\
\text { settings from three } \\
\text { facilities }\end{array}$ & $\begin{array}{l}\text { OTs } \\
\mathrm{n}=19 \\
\text { Multidisciplinary } \\
\text { stakeholders } \\
\mathrm{n}=8\end{array}$ & $\begin{array}{l}\text { Intervention } \\
\text { Pre-discharge } \\
\text { home visit }\end{array}$ & $\begin{array}{l}\text { To understand both occupational therapists' and } \\
\text { multidisciplinary stakeholders' perceptions and } \\
\text { contemporary practice regarding decision-making and } \\
\text { pre-discharge home visits through exploration of } \\
\text { experience and current practice in the Australian } \\
\text { context. Investigation of factors associated with when, } \\
\text { how and to whom pre-discharge home visits are provided }\end{array}$ & n.a. & \\
\hline $\begin{array}{l}\text { Hibberd, } \\
2008 \text { [36] }\end{array}$ & $\begin{array}{l}\text { Semi-structured } \\
\text { interview } \\
\text { UK } \\
\text { Intermediate care } \\
\text { unit }\end{array}$ & $\begin{array}{l}\text { Patients } \\
\mathrm{n}=4 \\
65 \text { years and } \\
\text { older } \\
\square 50 \%\end{array}$ & $\begin{array}{l}\text { Intervention } \\
\text { Predischarge home } \\
\text { visit / access visit }\end{array}$ & $\begin{array}{l}\text { Part of an evaluation study; } \\
\text { - To gain patient perspectives on home visiting process - } \\
\text { to ensure service meets needs }\end{array}$ & n.a. & n.a. \\
\hline $\begin{array}{l}\text { Money et } \\
\text { al., } 2015 \\
{[10]}\end{array}$ & $\begin{array}{l}\text { Semi-structured } \\
\text { interviews, } \\
\text { thinking aloud } \\
\text { UK } \\
\text { Community } \\
\text { dwelling }\end{array}$ & $\begin{array}{l}\text { Community } \\
\text { dwelling older } \\
\text { people } \\
n=10 \\
56-80 \text { years } \\
50 \%\end{array}$ & $\begin{array}{l}\text { Intervention } \\
\text { Virtual reality } \\
\text { predischarge home } \\
\text { assessment with } \\
\text { interior design } \\
\text { application }\end{array}$ & $\begin{array}{l}\text { - To explore community-dwelling, older adults' } \\
\text { perceptions of using a computerized 3D interior design } \\
\text { application (perceived usefulness, ease of use, and } \\
\text { actual use) } \\
\text { - To consider the potential barriers and opportunities of } \\
\text { using CIDA as an assistive tool within the predischarge } \\
\text { home visits process }\end{array}$ & n.a. & n.a. \\
\hline $\begin{array}{l}\text { Nygard et } \\
\text { al., } 2004 \\
{[37]}\end{array}$ & $\begin{array}{l}\text { Interviews, focus } \\
\text { groups, } \\
\text { participant } \\
\text { observation } \\
\text { Sweden }\end{array}$ & $\begin{array}{l}\text { Patients } \\
\mathrm{n}=23 \\
78 \text { years } \\
\text { (range } 68-86 \text { ) } \\
\square 50 \% \\
\text { Living alone } \\
\mathrm{n}=12 \\
\text { OTs }\end{array}$ & $\begin{array}{l}\text { Intervention } \\
\text { Single } \\
\text { predischarge home } \\
\text { visit }\end{array}$ & $\begin{array}{l}\text { - To describe and illustrate, from both clients' and } \\
\text { therapists' perspectives, the occupational therapy } \\
\text { interventions and recommendations that were } \\
\text { undertaken and followed-up in common practice during } \\
\text { predischarge home visits; } \\
\text { - To gain insight in the accuracy of expectations of } \\
\text { therapists and in perceived usefulness of predischarge } \\
\text { home visits to clients }\end{array}$ & n.a. & n.a. \\
\hline
\end{tabular}

Page $7 / 20$ 


\begin{tabular}{|c|c|c|c|c|c|c|}
\hline & $\begin{array}{l}\text { Geriatric acute } \\
\text { care, mixed } \\
\text { diagnoses }\end{array}$ & $\mathrm{n}=9$ & & & & \\
\hline $\begin{array}{l}\text { Threapleton } \\
\text { et al., } 2017 \\
{[3]}\end{array}$ & $\begin{array}{l}\text { Semi-structured } \\
\text { interview } \\
\text { UK } \\
\text { Acute care, } \\
\text { rehabilitation, } \\
\text { community, } \\
\text { stroke }\end{array}$ & $\begin{array}{l}\text { Patients } \\
\mathrm{n}=8 \\
68 \text { years } \\
\text { (range } 44-92 \text { ) } \\
\square 75 \% \\
\text { Stroke survivors } \\
\mathrm{n}=4 \\
70 \text { years } \\
\text { (range } 61-79 \text { ) } \\
\text { 口 } 75 \% \\
\text { OTs } \\
\mathrm{n}=13\end{array}$ & $\begin{array}{l}\text { Intervention } \\
\text { Virtual } \\
\text { predischarge home } \\
\text { visit }\end{array}$ & $\begin{array}{l}\text { - To explore perceptions concerning the acceptability, } \\
\text { potential utility and limitations of the use of a virtual } \\
\text { reality interior design application from the perspectives } \\
\text { of therapists and patients }\end{array}$ & n.a. & n.a. \\
\hline $\begin{array}{l}\text { Whitehead } \\
\text { et al., } 2014 \\
\text { [38] }\end{array}$ & $\begin{array}{l}\text { Semi-structured } \\
\text { interview } \\
\text { UK } \\
\text { Acute, } \\
\text { rehabilitation, } \\
\text { mixed, hyper } \\
\text { acute, stroke }\end{array}$ & $\begin{array}{l}\text { OTs } \\
\mathrm{n}=20\end{array}$ & $\begin{array}{l}\text { Intervention } \\
\text { Predischarge home } \\
\text { assessment visits }\end{array}$ & $\begin{array}{l}\text { - To explore what factors occupational therapists } \\
\text { consider when deciding which patients with a stroke } \\
\text { need a predischarge home assessment visit }\end{array}$ & n.a. & n.a. \\
\hline
\end{tabular}

\section{Setting and participants}

The studies were published between 2002 and 2020, and the majority were conducted in the UK $[3,4,8,10,32-34,36,38,40]$ and Australia $[2,25-27,29-31$, 39]. One study each was carried out in Germany [9], France [28] and Canada [35], and two studies were conducted in Sweden [23, 24, 37].

Participants in RCTs were recruited in acute care settings $[2,4,9,23,24,26-30]$ and rehabilitation units $[8,25]$, and for qualitative studies in the community [10], in rehabilitation [35], in acute care [3, 32] and in intermediate care [36].

Diagnoses were mixed, not specified or not sufficiently reported in five RCTs [2, 9, 25-28, 30] and in seven qualitative studies [32-34, 36, 37, 39, 40]. In two RCTs and three qualitative studies, participants had suffered from a stroke $[3,4,8,35,38]$. The diagnosis was hip fracture in another RCT [23, 24].

The qualitative studies reported on participants' views [3, 31, 35-37] and on views of OTs [3, 32, 33, 35, 37, 38], families [31, 35], and older community-dwelling people [10].

\section{Types of interventions}

Interventions comprised a single predischarge home visit only $[4,8,25,26]$ as well as additional supportive interventions through in-hospital activities $[9,23$, $24,28]$, including extended assessment $[2,9,25]$ and / or extended training $[9,24]$. Further intervention components were patient education $[4,8,25,28]$ and post-discharge follow-ups [2, 9]. All the PDHAs were conducted by OTs alone, or with additional professionals allied to health care (physiotherapists, nurses, social workers) $[9,28]$. The patients were present during the home assessment in seven out of eight RCTs $[2,4,8,23-26,28]$. All but one of the interventions were conducted in the patient's home, and included functional assessment [9]. Virtual home visits, conducted at the hospital, were investigated in one study [4]. The intervention details are available from the corresponding author.

\section{Types of comparators}

Usual care in Australia was described as an in-hospital access to multidisciplinary care [26], as well as a structured interview with the 0T, including two structured assessments and an access visit if more information was required, such as measurements for rails [2] or additional patient education and information about equipment use and community services [25]. Usual care in the UK was described as structured interviews and general discussions about potential problems, and referring to agencies [8]. One study [4] reported additional home / access visits as a control, if required. Usual care in Sweden [23, 24] comprised nursing care and instruction from a physiotherapist for walking aids. Usual care in Germany [9] comprised comprehensive geriatric assessment and recommendations. Usual care in France was not described [28].

\section{Risk of bias within studies}

The results of the risk of bias assessment are summarized in Figure 2 and are presented in more detail in Additional File 3 . Risk of selection bias was low in all but one study, where it was unclear [28]. For the outcome IADL/ADL, the risk of performance bias was unclear in five studies [2, 4, 8, 26, 28], and high in two of the seven studies addressing this outcome. For quality of life, the risk of performance bias was high in two studies [23-25] and unclear in three of five studies addressing this outcome $[4,8]$. Risk of readmission and risk of falling were not biased in all six studies addressing this outcome [2, 8, 9, 23-26, 28]. Mobility was detected in two studies with a low or unclear performance bias, respectively. Three studies assessed fear of falling with a high or unclear risk of bias, respectively $[4,25,26,28]$. Risk of detection bias was unclear in two studies $[23,24,28]$. Risk of attrition bias was high in one study [23, 24]. Risk of other bias was unclear in one study [2].

The quality appraisal of the qualitative studies is shown in Additional File 4 . The quality of the studies did not influence the analysis since all the studies were considered as being valuable for our research question. 


\section{Effectiveness of PDHA versus usual care}

Eight RCTs including 1149 participants compared PDHA with usual care [2, 4, 8, 9, 23-26, 28]. Forest plots for comparisons are displayed in Additional File 6. Meta-analysis was performed for Instrumental Activities of Daily Living (IADL) and Activities of Daily Living (ADL), quality of life (Qol), mobility, fear of falling, risk of falling and risk of readmission. Details on the GRADE judgment are reported in Additional File 5.

The summary of findings for the main outcomes is presented in Table 2.

Assessment of reporting bias through funnel plot analysis was not appropriate due to the small number of studies.

IADL/ADL (Instrumental) Activities of Daily Living (IADL/ADL) were measured in seven of eight studies on patients with stroke, hip fractures, or mixed or unspecified diagnoses respectively [2, 4, 8, 23-26, 28]. Five studies used the Extended Activities of Daily Living scale (NEADL) [41], another used the Functional Autonomy Measurement System (SMAF) [42], each as a full questionnaire. One study used the subscale Physical Function from the Swedish Health-Related Quality of Life Survey (SWED-QUAL), which assesses a patient's ADL performance (e.g. dressing, climbing stairs) and is therefore comparable to the content of included ADL-measures [43]. There was no overall effect in (instrumental) functions of daily living for participants at the latest follow-up after receiving PDHA when measured with various scales (655 participants, SMD $-0.17,95 \% \mathrm{Cl}[-0.87$ to 0.53$], p=0.64, I^{2}=91 \%$ ). The quality of evidence was judged to be very low due to concerns about risk of bias (blinding of outcome assessment), inconsistency and imprecision with considerable heterogeneity. $\mathrm{A}$ sensitivity analysis of five studies using the same scale (NEADL) confirmed the results (MD -0.32 [ -1.26 to 0.61$], p=0.50, I^{2}=0 \%$ ) with very low heterogeneity [2, 4 , 8]. GRADE assessment indicated low quality due to high risk of bias (blinding of outcome assessment) and imprecision.

Quality of life (QoL) Three studies used the EQ-5D overall score [44] and another three the subscales of the EQ-5D measure of health status from the EuroQol Group (EQ-5D) or SWED-QUAL [43], respectively. Pooling all studies with any Qol measure [4,23-25] showed no statistically significant group differences of PDHA compared to usual care for patients with stroke, hip fractures, or mixed diagnoses respectively, with moderate heterogeneity (263 participants, SMD $0.06,95 \% \mathrm{Cl}[-0.30$ to 0.42$], p=0.74, I^{2}=42 \%$ ). Applying the GRADE approach, we assessed the quality of the evidence to be very low due to a risk of bias (unblinded participants and personnel) and imprecision of results. A sensitivity analysis of three studies using the same scale (EQ-5D overall score) did not significantly affect the Qol outcome (186 participants MD $0.03,95 \% \mathrm{Cl}[-0.08$ to 0.15$], p=0.56, I^{2}=0 \%$ ). The quality of the evidence for these results was low due to inconsistency and imprecision.

Mobility. Two studies assessed mobility through Performance-Oriented Assessment of Mobility Problems (Tinetti) or The Rivermead Mobility Index (RMI) rating scale for patients with mixed diagnoses or stroke, respectively $[4,25]$ Pooling these studies showed no improvement at the latest time points of followup at one and three months (26 participants, SMD $1.24,95 \% \mathrm{Cl}[-0.69$ to 3.17$], p=0.21,12=78 \%$ ). However, the quality of the evidence was rated very low due to inconsistency and high imprecision based on a very small number of participants with high heterogeneity.

Three studies measured fear of falling in participants with a stroke or mixed diagnoses, respectively, using the Falls Efficacy Scale - International (FES-I) [47]. There might be a slight trend towards an increase in fear of falling in participants who received the PDHA intervention. Applying the fixed effect Model (FEM) resulted in a statistically significant effect in favor of the control group (85 participants, MD $-4.7495 \% \mathrm{Cl}[-8.30$ to -1.18$]$, $\mathrm{p}=0.002$ ) with moderate heterogeneity $\left(I^{2}=51 \%\right)$. When a pre-specified random effects model (REM) was used, there was no difference between groups in pooled effects for fear of falling (85 participants, MD $-4.01,95 \% \mathrm{Cl}[-10.4,2.05], \mathrm{p}=0.51)$ with moderate heterogeneity $\left(\mathrm{I}^{2}=51 \%\right)$. Using the GRADE approach, we assessed the quality of the evidence for this outcome to be very low due to a risk of performance bias (unblinded participants and personnel) and imprecision of results resulting from the very small number of participants.

Risk of falling The overall effect of PDHA on reducing risk of falling was not statistically significant (523 participants, RR $0.88,95 \% \mathrm{Cl}[0.69$ to 1.13$]$, $\mathrm{p}=0.32$ ), $\left.\mathrm{I}^{2}=0 \%\right)$. Included were patients with mixed or unspecified diagnoses, respectively, hip fractures and stroke $[8,9,25,26,28]$. The quality of evidence was assessed as moderate because considerable harm and benefit were included in the confidence intervals of all the studies. We were therefore concerned with regard to imprecision.

Risk of readmission Pooling five studies showed no statistically significant effect on the reduction of readmissions throughout an average of 5 months after receiving PDHA (590 participants, RR $1.09,95 \% \mathrm{Cl}[0.64$ to 1.87$], \mathrm{p}=0.70, \mathrm{I}^{2}=43 \%$ ) in patients with unspecified or mixed diagnoses or stroke, respectively [2, 8 , $25,26,28]$. Applying the GRADE approach, the quality of evidence was assessed as moderate because significant harm and benefit were included in the confidence intervals of all the studies. For this reason, we were concerned with regard to imprecision.

\section{Outcomes from single studies:}

Overall independence was assessed with the Modified Ranking Scale [48] in one study with a missing significant difference between the groups at one month after discharge (16 participants, MD $-0.2095 \% \mathrm{Cl}[-0.65$ to 0.25$], \mathrm{p}=0.38$ ) [4].

\section{Psycho-social outcomes}

One study reported on three different psycho-social outcomes, although all had missing significant differences at one month after discharge: Emotional distress in medical settings was measured through the GHQ-28 $[49,50]$ in 85 participants (in the intervention group with median 19; IQR 12.25-23.75 vs. median 23; IQR 15.5-31.5 in the control group; p=0.10). Depression was measured through The Stroke Aphasic Questionnaire [51] in 85 participants (in the intervention group with median 6; IQR 3.25-9.75 vs. median 7; IQR 4-11 in the control group; $p=0.37$ ). Caregiver strain was measured though the Caregiver Strain Index [52] in 85 participants (in the intervention group with median 5.5; IQR 1.75-7 vs. median 6; IQR 5-8 in the control group; p=0.11). 


\section{Process outcomes}

The number of recommendations was reported in two studies with significant increases in the number of modifications in the intervention group compared to the control group at 30 or 90 days after discharge, respectively (average number of modifications 2.8 ( 1.6 to 3.9 ), p <.001 in one study and range $0-13$ in intervention vs. $0-7$ in controls, $p=0.001$ in another study) [2].

\section{Admissions to hospitals and care facilities}

The number of emergency department visits was reported in one study with missing significant differences between the groups at 90 days after discharge (337 participants; $\mathrm{RR}=1.06,95 \% \mathrm{Cl}[0.73$ to 1.55$], \mathrm{p}=0.73$ [2].

One study (86 participants) reported missing significant differences in the number of institutionalizations after 12 months (60 participants, RR= 0.58 ; $95 \% \mathrm{Cl}$ 0.26 to $1.27 ; p=0.17)$ [28].

The number of patients receiving community support was reported in one study, which stated that, three months after discharge, a total of three patients across groups received community support (seven patients across groups received support at baseline) [25].

Table 2: Summary of findings

\section{PDHA compared with usual care for adults with any diagnosis at all}

Patients or population: adults with any diagnosis at all (except mental disorders only)

Setting: acute / sub-acute hospital care or rehabilitation unit

Intervention: predischarge home assessment

Comparison: usual care

Outcomes

IADL/ADL. Various scales. Including studies with NEADL,

NEADL (60), SMAF, SWED-QUAL Subscale Physical function.

Higher score indicates better function. Mean duration of follow-

up: 8 months (range 1-12 months)

IADL/ADL. NEADL Score 0-22 points. Higher score indicates better results. Mean duration of follow-up: 2.8 months (range 16 months)

Quality of life. Various scales: EQ-5D overall score, EQ-5D

subscale VAS, SWED-QUAL subscale general health perception.

Higher score indicates better health status. Mean duration of

follow-up: 2.6 months (range 1-6 months)

Quality of life. EQ-5D overall score 0-1. Higher score indicates

better health status. Mean duration of follow-up: 2.6 months (range 1-6 months)

Mobility. Various scales: Tinetti (scale 4-24) and RMI (0-15). Higher scores indicate better mobility. Mean duration of followup: 2 months (range 1-3 months)

Fear of falling. FES-I Score 10-100. Higher scores indicate more confidence. Mean duration of follow-up: 3.3 months (range 1-6 months)

\begin{tabular}{|c|c|c|c|}
\hline $\begin{array}{l}\text { SMD* or MD* or } \\
\text { RR, [95\% CI], } \\
\mathrm{I}^{2}, \mathrm{p}\end{array}$ & $\begin{array}{l}\text { Number of } \\
\text { participants } \\
\text { (number of } \\
\text { studies) }\end{array}$ & GRADE & Comments \\
\hline $\begin{array}{l}S M D-0.17[-0.76, \\
0.42], I^{2}=90 \% \\
p=0.58\end{array}$ & $655(7)$ & $\begin{array}{l}\text { ФOOO } \\
\text { very lowa }\end{array}$ & \\
\hline $\begin{array}{l}M D-0.35[-1.31, \\
0.61], I^{2}=79 \% \\
p=0.34\end{array}$ & $510(5)$ & $\oplus O O O$ very $l o w^{c}$ & \\
\hline $\begin{array}{l}S M D 0.06[-030, \\
0.42] I^{2}=42 \% \\
p=0.74\end{array}$ & $263(5)$ & $\underset{l o w^{c}}{\oplus O O O}$ & \\
\hline $\begin{array}{l}M D 0.03[-0.08 \\
0.15], I^{2}=0 \% \\
p=0.56\end{array}$ & $186(3)$ & $\begin{array}{l}\oplus \oplus O O \\
l o w^{b}\end{array}$ & \\
\hline $\begin{array}{l}S M D 1.24\left[{ }^{\prime}-0.69,\right. \\
3.17], I^{2}=78 \% \\
p=0.21\end{array}$ & $26(2)$ & $\oplus O O O$ very $l o w^{b}$ & \\
\hline $\begin{array}{l}M D-4.01[-10.4 \\
2.05], I^{2}=51 \% \\
p=0.19\end{array}$ & $85(3)$ & $\oplus O O O$ very low ${ }^{c}$ & $\begin{array}{l}\text { Fixed } \\
\text { effect } \\
\text { model: } \\
\text { (MD -4.74 } \\
\text { [-8.30, } \\
-1.18] \\
\text { I2=51\%, } \\
\text { p=0.009 } \\
\end{array}$ \\
\hline $\begin{array}{l}R R 0.88[0.70, \\
1.09], I^{2}=0 \% \\
p=0.25\end{array}$ & $501(5)$ & $\oplus \oplus \oplus O^{d}$ moderate & \\
\hline $\begin{array}{l}R R 1.09[0.64 \\
1.87], I^{2}=43 \% \\
p=0.74\end{array}$ & $590(5)$ & $\oplus \oplus \oplus O^{d}$ moderate & \\
\hline $\begin{array}{l}\text { Zero adverse } \\
\text { events in both } \\
\text { groups were } \\
\text { reported in one } \\
\text { study. }\end{array}$ & $59(1)$ & & \\
\hline
\end{tabular}




\section{Qualitative synthesis}

Based on four comprehensive descriptive themes, seven analytical themes were identified regarding the barriers and facilitators of the PDHA process. Details are reported in Additional File 7 (Summary of the descriptive themes) and Additional File 8 (Overview on analytical themes).

\section{Barriers and facilitators in PDHA process, analytical themes}

The safety assessment of the home environment. Participants highlighted the importance of safety after hospital discharge [40]. The aim was to identify any required provisions and adaptations before going home and to identify and eliminate risks within the home $[3,32,34,40]$, as well as to assess whether the home environment was suitable for the required equipment [34]. The facilitators were the structured identification of risk factors and patient/family's awareness of these factors following education, therefore enabling practical recommendations [40]. VR was identified as a useful tool to educate patients in order to identify and discuss risk factors, thus increasing patients' awareness [3]. Therefore, we inferred the implication: 1 "Use environmental assessments together with patients to provide education about hazards."

Functional assessment of the patient at home as a reality check. The aim of a functional assessment in general was to assess whether the patient is able to manage within his / her home [34]. On the whole, the predischarge home visit was a chance for therapists to gain a realistic view of the patients' functions [34, 35, 37-40]. But this also applied vice versa: "It's making them [the patients] aware of that impact and how they might be able to overcome the problems they will encounter. [...] We do get patients who say 'Oh, once I'm home I'll be fine..., butIdont think theyllalwaysap $\prec$ iatethe lim itationstheyre going to encounter." [34]. PDHA gave information on future therapy sessions and helped to tailor individual rehabilitation goals [35, 37, 39]. Visiting their home motivated patients to do the therapy so that they could return home [32, 34, 35, 37, 40]. Performance tests at home can cause the patients to become anxious about failure, so the social skills of OTs are definitely needed [32,34]. However, the preparations for the functional requirements for carrying out activities of daily living at home in the context of PDHA offered the chance to reduce anxiety [40]. We inferred the implications: 2 Conduct a functional assessment that includes the living reality of the patient and helps the patient to find individual participation goals for therapy, and 2.1 Consider potential patient anxieties regarding the assessment situation.

Intervention planning and evaluation. Novice therapists in particular struggled with the aim and content of PDHA [40]. The actual timing of PDHA was highly dependent on organizational factors and resource availability $[34,35,39]$. There were often pragmatic aspects, like the availability of supportive network, patients' preferences or "gut feeling" to consider when deciding about whether or not to conduct a PDHA [37-40]. Working with community players often led to dissatisfaction with devices [37], without having the chance to follow up with the patient [39]. The facilitators were identified as: clear aims and assessment tasks, early patient identification and planning and a decision support tool [39], further use of standardized protocols during PDHA and collaboration with community services [40], as well as a formal evaluation after the PDHA [35]. The use of a digital interface to transmit environmental information could encourage the communication between the various stakeholders [10]. Therefore, we derived the implication 3: Use standardized procedures and materials to guide the PDHA process. Digital solutions might support the collaboration between hospital and community service providers.

Patient information about the home assessment procedure. Older people felt insufficiently informed prior to and after the home visit. Lack of information about the aims, the outcomes and the next steps of the process of PDHA made them feel insecure and anxious and excluded from the process [32, 34, 36]. Even during the home visit, there were situations in which the carer, but not the patient, was included in the process [32]. Written information about PDHA was seen as a facilitator by patients [35,36]. Some patients and therapists felt a lack of real informed choice about the assessment [32]. Therefore we derived the implication 4. Provide adequate (verbal and written) patient information about aim, process, assessment, results and consequences of the predischarge home assessment.

Patients' and family carers' acceptance of home modifications and aids. The concerns of the patients that the OT's modifications might hinder them in performing ADL in the usual and preferred way was identified as a barrier [32]. The use of a patient's know-how on where to use an aid most effectively in their home environment was a facilitator for acceptance [37]. From the OT's view it was seen as challenging to propose and communicate potential adaptations to the patients [10]. The lack of imagination regarding home modifications and adaptations [3] was seen as a barrier for acceptance. OTs and older people estimated that the use of visualization with a 3-D interior design software application would enable patients to better understand assistive technologies and adaptations [10,33]. OTs considered a virtual reality tool as superior to drawings and photographs [33]. Consequently, a more clear visualization was seen as a facilitator for OTs to communicate better about modifications or even explain better decisions against a patient bbeeing discharged to his/her own home [10]. In addition, a clear visualization as a joint basis for discussion was seen as a facilitator to include patients in the decisions about home modifications and aids, giving them a chance to give immediate feedback on proposed changes, thus leading to shared decision-making [3, 10, 33] . Therefore we inferred implication 5: Provide tailored adaptations based on shared decision-making and involve explicitly patients' ideas, solutions and expectations in planning home modifications, and 5.1 Provide appropriate visualization for discussing recommended aids and home modifications.

Matching PDHA and clinical patient conditions. Different patient conditions in terms of diagnosis and related kinds of impairments as well as the levels of impairment may be factors that facilitate or inhibit the performance of certain PDHA approaches. For example, sensory and visual limitations might be an indication for a home visit. However, the same limitations may have an adverse effect on the use of a virtual home assessment. Too low or too high levels of functional limitations spoke rather against home visits and in favor of ward-based assessments or access visits. Summing up, different patient conditions required different approaches for assessment. We inferred implication 6: Tailor the intervention components and mode of delivery to patients' level and kind of impairments.

Context factors in daily routine of PDHA. Many of the qualitative studies identified factors that may have a beneficial or impeding effect on the decision of whether and how to conduct PDHA. Lack of resources (staff, time, secretarial backup, technical resources for virtual assessment) hampers the process of organization and execution $[3,32,34,36,38,39]$. A virtual approach to PDHA could partially overcome some of the obstacles (e.g. out of hospital catchment 
zone, car availability, safety requirements for allowing a home visit with patient) [33]. Factors such as risks while making home visits and the organization of appropriate PDHA attendants have an impact on the process of PDHA [34, 37, 39, 40]. Therefore, we derived the implication 7: Consider specific context factors in PDHA-design.

\section{Integrative synthesis}

An overview of the results of the analysis at the individual study level with regard to the respective qualitative results (whether the PDHA intervention had considered implications 1-7) and the outcome effects in the patient outcomes is shown in table 3.

Table 3. Synthesis of practice implications and RCT interventions 
Integrative synthesis of qualitative and quantitative results in studies in PDHA

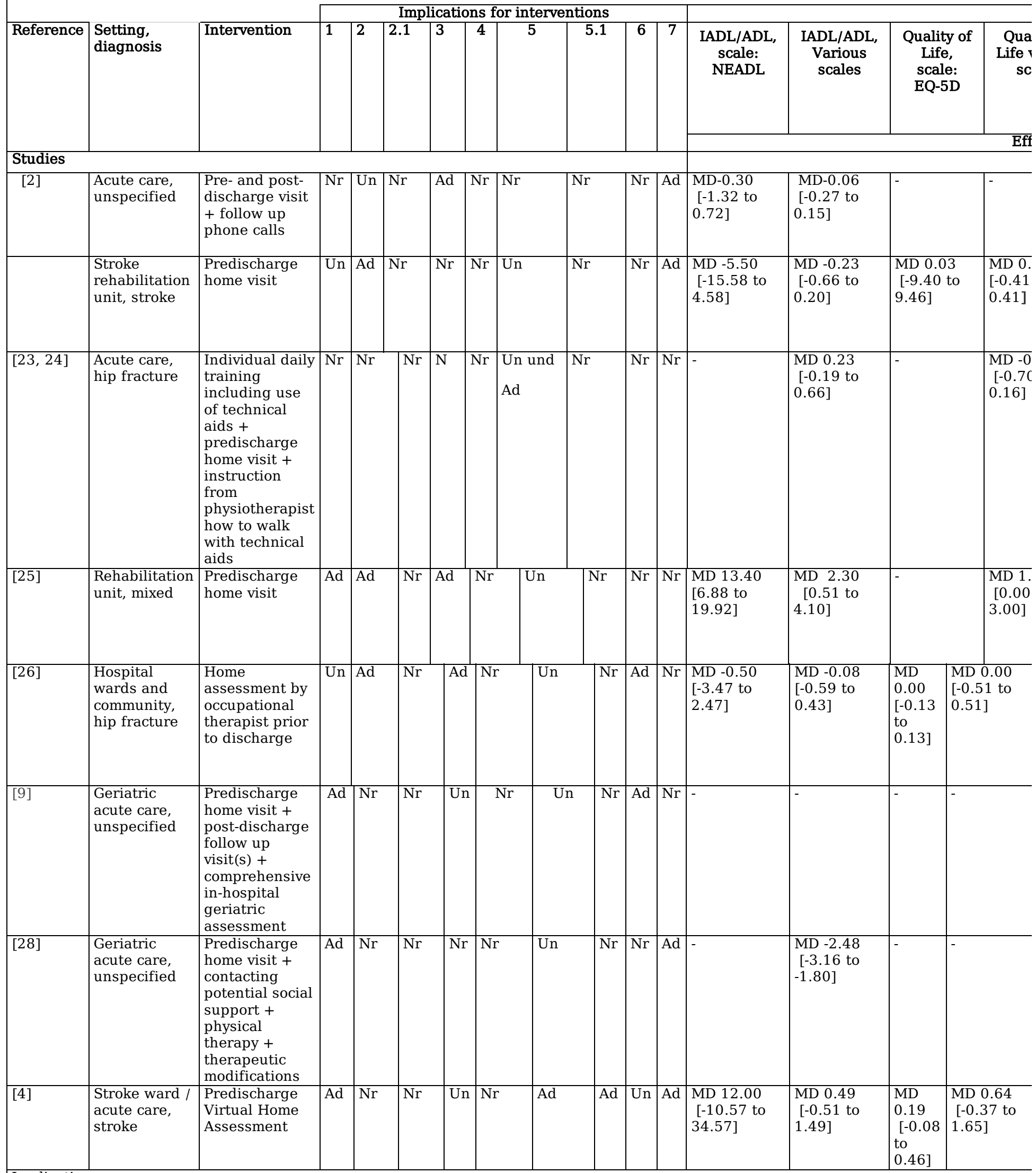

\section{Implications:}

1) Use environmental assessments together with patients to provide education about hazards. 2) Conduct a functional assessment that includ individual participant goals for therapy. 2.1) Consider potential patient anxieties regarding the assessment situation. 3) Use standardized proc could support the collaboration between hospital and community service providers. 4) Provide adequate (verbal and written) patient informatic predischarge home assessment. 5) Provide tailored adaptations based on shared decision-making and involve explicitly patient's ideas, solutiou appropriate visualization for discussing recommended aids and home modifications. 6) Tailor the intervention components and mode of deliver context factors in PDHA-design. 


\section{Discussion}

This review investigated the impact of PDHA on functional outcomes associated with a successful return to community living for patients with various diagnoses. It also identified barriers and facilitators of the PDHA process from which recommendations for clinical practice could be derived.

\section{Improving patient outcomes with PDHA}

Overall, there is a very low to moderate quality of evidence that PDHA might not result in any difference in patient outcomes when compared to usual care. There were only a few studies, and each of them investigated a variety of outcomes.

PDHA seems to have no impact on the quality of life. This result is in line with the systematic review by Lockwood et al. [5]. Although we included two additional RCTs and excluded one cohort study, our analysis also showed only a small overall effect size in favor of PDHA. However, the quality of the evidence for this is very low to low. Further studies with a robust sample size are required that are powered to assess effects in quality of life as a primary outcome.

Mobility To our knowledge, the evidence on the effect of PDHA on mobility was assessed for the first time in our review. Since there were only two studies with different outcome measures and very small sample sizes, the quality of evidence is very low about an effect of PDHA on mobility.

Risk of falling Since we only included randomized trials and excluded cohorts, our results do not confirm any effects of PDHA on risk reduction for falls in contrast to the findings of Lockwood [26]. The quality of the evidence for this finding is moderate. The few included studies reported conflicting results with large confidence intervals, so the body of evidence is still unclear. Therefore, further research is needed to confirm a possible effect.

Fear of falling To our knowledge, the evidence on the effect of PDHA on the fear of falling was assessed for the first time in our review. Our finding regarding the fear of falling is contrary to the evidence of an effect of PDHA on the reduction of falls, which Lockwood et al had found. Our finding indicated that the fear of falling increases to a small extent. However, there is only low quality of evidence from two small studies showing a slight but significant increase of fear of falling in the intervention group when compared to usual care. This is in contrast to existing literature, which assumes that an increased fear of falling contributes to an increased risk of falling [53]. Our findings might be explained by the fact that an element of PDHA is to increase patients' awareness of the potential risk of falling at home, which might also result in an increased fear of falling. This needs further investigation and should be considered when conducting the PDHA and the measures of discharge planning that result from the PDHA.

IADL/ADL When pooling various IADL measures and ADL measures of seven RCTs together, there was no effect of PDHA on IADL/ADL. Pooling only the studies that used the NEADL measure also resulted in no effect of PDHA. The quality of the evidence of our analysis is low although we added three additional RCTs, comparing to an earlier review by Lockwood et al. [5]. These authors suggested a benefit in ADL, also with a low quality of the evidence. These differences can be explained by the different approaches used: Lockwood et al. made a distinction between activity and participation measures. Since these constructs are very closely related at the level of measures for ADL, we decided not to differ between activity and participation measures. Since improving independence in everyday living is a core objective of PDHA, these results seem surprising. One reason might be the appropriateness of the chosen outcome measures used in RCTs [54]. The outcome measures included a range of items that are unlikely to be affected by PDHA interventions (e.g., items related to various activities outside the living environment or items for the assessment of communication functions or mental functions). A definition of desirable activity and participation items that are operationalized for each individual patient could make a measurement more sensitive and thus make changes more visible [54]. Standardized measures for patient goal attainment (e.g., The Canadian Occupational Performance Measure, COPM [55]) or single items from validated ADL scales would be conceivable here. At the same time, such measures would enhance patient involvement, which is believed to be fundamental to occupational therapy practice and the discharge planning process $[56,57]$.

Our findings on the effects of PDHA on risk of readmission for people who received a PDHA intervention did not show any difference compared to patients who received usual care. This result is in line with the existing literature [5]. Since we included an additional RCT with a large sample size, while excluding the cohort study, the quality of the evidence increased from "low" according to Lockwood et al. to "moderate". A PDHA is expected to reduce risk of readmission to the hospital by preventing falls and their consequences. This was probably the reason why the majority of study authors chose this outcome measure for their RCTs. However, there are a number of other events causing readmission to hospital, which cannot be affected by PDHAs (e.g., relapse or aggravation of a previously known condition, complications and drug-related adverse events [58]). Therefore, risk of falling and fall-related consequences might be more appropriate outcome measures for assessing the effects of PDHA than readmission to hospital.

In this review, we identified potential factors for effectiveness from the views of stakeholders involved in the PDHA process; his method showed that some clear recommendations for practice could be developed systematically.

We included seven additional qualitative studies in the analysis of the barriers and facilitators of the PDHA process compared to earlier syntheses [5, 11]. Various implications were derived from the analysis of the qualitative studies as being meaningful criteria for PDHA design and implementation. These criteria - the necessity of considering the identification of hazards, the functional assessment in the context of the real home environment, and the inclusion of the patient's participation goals and priorities in the assessment - are congruent with those criteria used in a previous study [59]. While this earlier review on environmental interventions defined these criteria from the best practice view of therapists, we were able to derive them systematically from the perspectives of therapists, patients and relatives. In addition, we were able to identify clinical factors influencing the execution of PDHA.None of the included RCTs addressed all of the abovementionedmeaningful aspects of intervention design. This illustrates that a modification of PDHA to improve patient-centeredness is indicated and might explain the missing effects on the investigated outcomes.PDHAs themselves fulfill all the characteristics of complex interventions, 
especially when a PDHA is part of the discharge management (e.g., involving a variety of stakeholders, organizational levels and outcomes) [7, 20]. Nevertheless, none of the included studies reported to have taken into account the current recommendations for the development of complex interventions [20]. Rather than the evaluation of existing PDHA approaches, an adaptation of PDHA interventions is needed, including a sound description of the context, the consequent inclusion of the user perspective as well as the current evidence.

In this review, qualitative studies focusing on general home modifications, regardless of the setting in which they were conducted, were excluded. However, as an integral part of a PDHA intervention, the stakeholders' view of home modifications should also be examined in the future and the relevant implications for the design of PDHA should be derived.

\section{Limitations}

Our review has limitations owing to the shortcomings of the underlying studies. We decided to forgo subgroup analyses due to the small number of studies in this field. In order to take the heterogeneity of the studies into account a random effects model was used for the analysis. The experiences and beliefs of the participants in the included qualitative studies were shaped by the context of specific healthcare and insurance systems and may not be valid in other regions. The findings of the integrative synthesis must be interpreted with caution. First, the implications derived from the individual studies by the thematic analysis have to be considered on the background of their particular study samples in their respective context and therefore they lack generalizability. We tried to overcome this limitation by including views from different stakeholder groups and different contexts of PDHA in a thematic analysis and distinguished between the perspectives of the different stakeholder groups. Second, in some studies it might not be possible to distinguish whether the implications were ultimately not taken into account during the implementation, or whether they were only insufficiently reported. Our comprehensive search strategy minimized the risk of missing studies, as we searched through the reference lists of systematic reviews, conducted a forward citation search and searched trial registers. A language bias due to the English and German language restriction cannot be ruled out. Further valuable strengths of our review include an unlimited search period and the screening and critical appraisal by two independent scientists.

\section{Conclusions}

This systematic review revealed very low to moderate quality of evidence that PDHAs might have no impact on patient outcomes that are associated with a successful return to community living. Therefore, no conclusion can be drawn as to whether PDHA should be performed or to what kind of PDHA is required. However, implications in intervention design can be drawn from qualitative studies of stakeholders' perspectives on facilitators and barriers in the process of PDHA. Current RCTs partially consider these implications for complex PDHA interventions. For future PDHA, careful intervention development should be based on the existing qualitative evidence of stakeholder views. In future research, sufficiently robust RCTs using valid effect size estimates are needed in order to assess the effects of PDHAs. The use of appropriate outcome measures, reflecting the users' demands on the PDHA process as well as the individual character of the patients' adjustment to function and home environmental requirements, might improve the evaluation of the interventions' effectiveness. Future studies should describe the PDHA intervention adequately, including how it is embedded in the discharge management to improve the dependability and to contribute to a better understanding of how the intervention might work.

\section{Abbreviations}

3D: three-dimensional; ADL/IADL: Activities of Daily Living/Instrumental Activities of Daily Living; COPM: Canadian Occupational Performance Measure; EQ5D: measure of health status from the EuroQol Group (EQ-5D); FE: Fixed Effect Model; FES-I: Falls Efficacy Scale-International; FU: follow-up; GRADE: The Grading of Recommendations Assessment, Development and Evaluation working group; MRS: Modified Ranking Scale; n.a.: not applicable; Nr: not reported; NEADL: Extended Activities of Daily Living scale; OT: Occupational Therapy; PDHA: Predischarge home assessment; RCT: Randomized Controlled Trial; RE: Random Effects Model; RMI: The Rivermead Mobility Index; SMAF: The Functional Autonomy Measurement System; SWED-QUAL: The Swedish HealthRelated Quality of Life Survey; VR: Virtual Reality, vs: versus

\section{Declarations}

\section{Ethics approval and consent to participate}

Not applicable. This research article was a systematic review summarizing previously published literature.

\section{Consent for publication}

Not applicable.

\section{Availability of data and materials}

The data published may be found in the original manuscripts cited in the list of references. The data extraction sheets from the RCTs and the qualitative studies are available from the corresponding author on reasonable request.

\section{Competing interests}


The authors declare that they have no competing interests.

\section{Funding}

No external funding.

\section{Authors' contributions}

SuS initially planned the review and wrote the protocol. KK conducted the initial search supervised by SS. UKH and KK conducted the critical appraisal and data extraction. The qualitative synthesis was conducted by UKH, assisted by KK. The meta-analysis and mixed method analysis were performed by UKH. All the authors took part in interpreting the study data. UKH wrote the drafts of the manuscript with support from SS. All the authors approved the final version of the manuscript and are accountable for all aspects of the work.

\section{Acknowledgements}

We would like to thank Professor Gabriele Meyer for her general support as well as for her advice regarding the finalization of the manuscript for publication. Many thanks to Vivienne Krause for her help with editing the manuscript with regard to the English language. Thanks to AJE for the expert editing of the final paper. Thanks to Thomas Nordhausen from RefHunter for supporting the literature search.

\section{Author details}

UKH is an occupational therapist and postgraduate research associate in health and nursing sciences. KK is a medical student. SuS is a physiotherapist and postdoctoral researcher in health and nursing sciences.

\section{References}

References

1. Nikolaus, T., Detterbeck, H., Gartner, U., Gnielka, M., Lempp-Gast, I., Renk, C., Suck-Röhrig, U., Oster, P., Schlierf, G.: Der diagnostische Hausbesuch im Rahmen des stationären geriatrischen Assessments (Diagnostic house call within the scope of inpatient geriatric assessment). Zeitschrift fur Gerontologie und Geriatrie 28(1), 14-18 (1995)

2. Clemson, L., Lannin, N.A., Wales, K., Salkeld, G., Rubenstein, L., Gitlin, L., Barris, S., Mackenzie, L., Cameron, I.D.: Occupational Therapy Predischarge Home Visits in Acute Hospital Care: A Randomized Trial. Journal of the American Geriatrics Society 64(10), 2019-2026 (2016). doi: 10.1111/jgs.14287

3. Threapleton, K., Newberry, K., Sutton, G., Worthington, E., Drummond, A.: Virtually home: Exploring the potential of virtual reality to support patient discharge after stroke. British Journal of Occupational Therapy 80(2), 99-107 (2017). doi: 10.1177/0308022616657111

4. Threapleton, K., Newberry, K., Sutton, G., Worthington, E., Drummond, A.: Virtually home: Feasibility study and pilot randomised controlled trial of a virtual reality intervention to support patient discharge after stroke. British Journal of Occupational Therapy 81(4), 196-206 (2018). doi: $10.1177 / 0308022617743459$

5. Lockwood, K.J., Taylor, N.F., Harding, K.E.: Pre-discharge home assessment visits in assisting patients' return to community living: A systematic review and meta-analysis. Journal of rehabilitation medicine 47(4), 289-299 (2015). doi: 10.2340/16501977-1942

6. Sim, S., Barr, C.J., George, S.: Comparison of equipment prescriptions in the toilet/bathroom by occupational therapists using home visits and digital photos, for patients in rehabilitation. Australian occupational therapy journal 62(2), 132-140 (2015). doi: 10.1111/1440-1630.12121

7. Welch, A., Lowes, S.: Home Assessment Visits within the Acute Setting: a Discussion and Literature Review. British Journal of Occupational Therapy 68(4), 158-164 (2005). doi: 10.1177/030802260506800403

8. Drummond, A.E.R., Whitehead, P., Fellows, K., Sprigg, N., Sampson, C.J., Edwards, C., Lincoln, N.B.: Occupational therapy predischarge home visits for patients with a stroke (HOVIS): results of a feasibility randomized controlled trial. Clinical rehabilitation 27(5), 387-397 (2013). doi:

$10.1177 / 0269215512462145$

9. Nikolaus, T., Bach, M.: Preventing Falls in Community-Dwelling Frail Older People Using a Home Intervention Team (HIT): Results From the Randomized Falls-HIT Trial. Journal of the American Geriatrics Society 2003(3), 300-305 (51)

10. Money, A.G., Atwal, A., Young, K.L., Day, Y., Wilson, L., Money, K.G.: Using the Technology Acceptance Model to explore community dwelling older adults' perceptions of a 3D interior design application to facilitate pre-discharge home adaptations. BMC medical informatics and decision making 15,73 (2015). doi: 10.1186/s12911-015-0190-2

11. Atwal, A., Spiliotopoulou, G., Plastow, N., McIntyre, A., McKay, E.A.: Older Adults' Experiences of Occupational Therapy Predischarge Home Visits: A Systematic Thematic Synthesis of Qualitative Research. British Journal of Occupational Therapy 75(3), 118-127 (2012). doi: 10.4276/030802212X13311219571701

12. Liberati, A., Altman, D.G., Tetzlaff, J., Mulrow, C., Gøtzsche, P.C., loannidis, J.P.A., Clarke, M., Devereaux, P.J., Kleijnen, J., Moher, D.: The PRISMA statement for reporting systematic reviews and meta-analyses of studies that evaluate health care interventions: explanation and elaboration. PLoS medicine 6(7), e1000100 (2009). doi: 10.1371/journal.pmed.1000100 
13. Tong, A., Flemming, K., Mclnnes, E., Oliver, S., Craig, J.: Enhancing transparency in reporting the synthesis of qualitative research: ENTREQ. BMC medical research methodology 12, 181 (2012). doi: 10.1186/1471-2288-12-181

14. Hoffmann, T.C., Glasziou, P.P., Boutron, I., Milne, R., Perera, R., Moher, D., Altman, D.G., Barbour, V., Macdonald, H., Johnston, M., Lamb, S.E., Dixon-Woods, M., McCulloch, P., Wyatt, J.C., Chan, A.-W., Michie, S.: Better reporting of interventions: template for intervention description and replication (TIDieR) checklist and guide. BMJ (Clinical research ed.) 348, g1687 (2014). doi: 10.1136/bmj.g1687

15. Thomas, J., Harden, A.: Methods for the thematic synthesis of qualitative research in systematic reviews. BMC medical research methodology 8,45 (2008). doi: 10.1186/1471-2288-8-45

16. Higgins, J., Green, S.: Cochrane Handbook for Systematic Reviews of Interventions Version 5.1.0 [updated March 2011]

17. Critical Appraisal Skills Programme (CASP). Qualitative Research Checklist.

18. Letts, L., Wilkins, S., Law, M., Stewart, d., Bosch, J., Westmorland, M.: Guidelines for Critical Review Form: Qualitative Studies (Version 2.0) (2007)

19. Wan, X., Wang, W., Liu, J., Tong, T.: Estimating the sample mean and standard deviation from the sample size, median, range and/or interquartile range. BMC medical research methodology 14(135) (2014)

20. Craig, P., Dieppe, P., Macintyre, S., Michie, S., Nazareth, I., Petticrew, M.: Developing and evaluating complex interventions: the new Medical Research Council guidance. BMJ (Clinical research ed.) 337, a1655 (2008). doi: 10.1136/bmj.a1655

21. Schünemann H, Brożek J, Guyatt G, Oxman A, editors.: GRADE handbook for grading quality of evidence and strength of recommendations. Updated October 2013. (2013)

22. Braun, V., Clarke, V.: Using thematic analysis in psychology. Qualitative Research in Psychology 3(2), 77-101 (2006). doi: 10.1191/1478088706qp063oa

23. Hagsten, B., Svensson, O., Gardulf, A.: Early individualized postoperative occupational therapy training in 100 patients improves ADL after hip fracture: a randomized trial. Acta orthopaedica Scandinavica 75(2), 177-183 (2004). doi: 10.1080/00016470412331294435

24. Hagsten, B., Svensson, O., Gardulf, A.: Health-related quality of life and self-reported ability concerning ADL and IADL after hip fracture: a randomized trial. Acta orthopaedica 77(1), 114-119 (2006). doi: 10.1080/17453670610045786

25. Lannin, N.A., Clemson, L., McCluskey, A., Lin, C.-W.C., Cameron, I.D., Barras, S.: Feasibility and results of a randomised pilot-study of pre-discharge occupational therapy home visits. BMC health services research 7, 42 (2007). doi: 10.1186/1472-6963-7-42

26. Lockwood, K.J., Harding, K.E., Boyd, J.N., Taylor, N.F.: Predischarge home visits after hip fracture: a randomized controlled trial. CLIN REHABIL 33(4), 681692 (2019). doi: 10.1177/0269215518823256

27. Lockwood, K.J., Harding, K.E., Boyd, J.N., Taylor, N.F.: Home visits by occupational therapists improve adherence to recommendations: Process evaluation of a randomised controlled trial. AUST OCCUP THER J 67(4), 287-296 (2020). doi: 10.1111/1440-1630.12651

28. Pardessus, V., Puisieux, F., Di Pompeo, C., Gaudefroy, C., Thevenon, A., Dewailly, P.: Benefits of home visits for falls and autonomy in the elderly: A randomized trial study. American Journal of Physical Medicine \& Rehabilitation(81), 247-252 (2002)

29. Provencher, V., Clemson, L., Wales, K., Cameron, I.D., Gitlin, L.N., Grenier, A., Lannin, N.A.: Supporting at-risk older adults transitioning from hospital to home: who benefits from an evidence-based patient-centered discharge planning intervention? Post-hoc analysis from a randomized trial. BMC GERIATR 20(1), 84ÔÇÉ (2020). doi: 10.1186/s12877-020-1494-3

30. Wales, K., Salkeld, G., Clemson, L., Lannin, N.A., Gitlin, L., Rubenstein, L., Howard, K., Howell, M., Cameron, I.D.: A trial based economic evaluation of occupational therapy discharge planning for older adults: the HOME randomized trial. CLIN REHABIL 32(7), 919-929 (2018)

31. Aplin, T., Jonge, D. de, Gustafsson, L.: Understanding the dimensions of home that impact on home modification decision making. Australian occupational therapy journal 60(2), 101-109 (2013). doi: 10.1111/1440-1630.12022

32. Atwal, A., McIntyre, A., Craik, C., Hunt, J.: Older adults and carers' perceptions of pre-discharge occupational therapy home visits in acute care. Age and ageing 37(1), 72-76 (2008). doi: 10.1093/ageing/afm137

33. Atwal, A., Money, A., Harvey, M.: Occupational therapists' views on using a virtual reality interior design application within the pre-discharge home visit process. Journal of medical Internet research 16(12), e283 (2014). doi: 10.2196/jmir.3723

34. Atwal, A., Spilliotopoulou, G., Stradden, J., Fellows, V., Anako, E., Robinson, L., McIntyre, A.: Factors influencing occupational therapy home visit practice A qualitative study. Scandinavian Journal of Occupational Therapy(21), 40-47 (2014)

35. Cameron, J.I., Bastawrous, M., Marsella, A., Forde, S., Smale, L., Friedland, J., Richardson, D., Naglie, G.: Stroke survivors', caregivers', and health care professionals' perspectives on the weekend pass to facilitate transition home. Journal of rehabilitation medicine 46(9), 858-863 (2014). doi: $10.2340 / 16501977-1854$

36. Hibberd, J.: The home-visiting process for older people in the in-patient intermediate care services. Quality Ageing Older Adults 9(1), 13-23 (2008). doi: $10.1108 / 14717794200800003$

37. Nygård, L., Grahn, U., Rudenhammar, A., Hydling, S.: Reflecting on practice: are home visits prior to discharge worthwhile in geriatric inpatient care? Scandinavian journal of caring sciences 18(2), 193-203 (2004). doi: 10.1111/j.1471-6712.2004.00270.x

38. Whitehead, P., Fellows, K., Sprigg, N., Walker, M., Drummond, A.: Who should have a pre-discharge home assessment visit after a stroke? A qualitative study of occupational therapist's views. British Journal of Occupational Therapy 77(8), 384-391 (2014)

39. Godfrey, M., Cornwell, P., Eames, S., Hodson, T., Thomas, T., Gillen, A.: Pre-discharge home visits: A qualitative exploration of the experience of occupational therapists and multidisciplinary stakeholders. AUST OCCUP THER J 66(3), 249-257 (2019). doi: 10.1111/1440-1630.12561

40. Davis, A.J., Mc Clure, P.: An exploratory study of discharge planning home visits within an Irish context - investigating nationwide practice and nationwide perspectives. IR J OCCUP THER 47(2), 95-113 (2019). doi: 10.1108/IJOT-10-2018-0015 
41. Nouri, F., Lincoln, N.: An extended activities of daily living scale for stroke patients. Clinical rehabilitation(1), 301-305 (1987)

42. Hebert, R., Guilbault, J., Desrosiers, J., Debuc, N.: THE FUNCTIONAL AUTONOMY MEASUREMENT SYSTEM (SMAF): A CLINICAL-BASED INSTRUMENT FOR MEASURING DISABILITIES AND HANDICAPS IN OLDER PEOPLE. Geriatrics Today: Journal of Canadian Geriatric Society(September), 1-7 (2001)

43. Brorsson, B., Ifver, J., Hays, R.D.: The Swedish Health-Related Quality of Life Survey (SWED-QUAL). Quality of life research : an international journal of quality of life aspects of treatment, care and rehabilitation 2(1), 33-45 (1993)

44. Rabin, R., Charro, F. de: EQ-5D: a measure of health status from the EuroQol Group. Annals of medicine 33(5), 337-343 (2001)

45. Tinetti, M.E.: Performance-Oriented Assessment of Mobility Problems in Elderly Patients. Journal of the American Geriatrics Society(34), 119-126 (1986)

46. Collen, F.M., Wade, D.T., Robb, G.F., Bradshaw, C.M.: The Rivermead Mobility Index: A further development of the Rivermead Motor Assessment. International Disability Studies 13(2), 50-54 (1991). doi: 10.3109/03790799109166684

47. Yardley, L., Beyer, N., Hauer, K., Kempen, G., Piot-Ziegler, C., Todd, C.: Development and initial validation of the Falls Efficacy Scale-International (FES-I). Age and ageing 34(6), 614-619 (2005). doi: 10.1093/ageing/afi196

48. Van Swieten, J.C., Koudstaal, P.J., Visser, M.C., Schouten, H.J.A., van Gijn, J.: Interobserver Agreement for the Assessment of Handicap in Stroke Patients. Stroke 19(5), 604-607 (1988)

49. Goldberg, D.P., Williams, P.: The users guide to the GHQ. (1988)

50. Sterling, M.: General Health Questionnaire - 28 (GHQ-28). Journal of Physiotherapy 57(4), 259 (2011). doi: 10.1016/S1836-9553(11)70060-1

51. Lincoln, N.B., Sutcliffe, L.M., Unsworth, G.: Validation of the Stroke Aphasic Depression Questionnaire (SADQ) for use with patients in hospital

52. Robinson, B.C.: Validation of a Caregiver Strain Index. Journal of Gerontology 38(3), 344-348 (1983). doi: 10.1093/geronj/38.3.344

53. Pena, S.B., Guimarães, H.C.Q.C.P., Lopes, J.L., Guandalini, L.S., Taminato, M., Barbosa, D.A., Barros, A.L.B.L.d.: F ear of falling and risk of falling: a systematic review and meta-analysis. Acta paul. enferm. 32(4), 456-463 (2019). doi: 10.1590/1982-0194201900062

54. Coster, W.J.: Making the best match: selecting outcome measures for clinical trials and outcome studies. The American journal of occupational therapy : official publication of the American Occupational Therapy Association 67(2), 162-170 (2013). doi: 10.5014/ajot.2013.006015

55. Law, M., Baptiste, S., McColl, M., Opzoomer, A., Polatajko, H., Pollock, N.: The Canadian occupational performance measure: an outcome measure for occupational therapy. Canadian journal of occupational therapy. Revue canadienne d'ergotherapie 57(2), 82-87 (1990). doi:

$10.1177 / 000841749005700207$

56. Mroz, T.M., Pitonyak, J.S., Fogelberg, D., Leland, N.E.: Client Centeredness and Health Reform: Key Issues for Occupational Therapy. The American journal of occupational therapy : official publication of the American Occupational Therapy Association 69(5), 6905090010p1-8 (2015). doi: 10.5014/ajot.2015.695001

57. Weiss, M.E., Bobay, K., bahr, S.J., Costa, L.L., Hughes, R.G.: A Model for Hospital Discharge Preparation: From Case Management to Care Transition

58. Yam, C.H.K., Wong, E.L.Y., Chan, F.W.K., Wong, F.Y.Y., Leung, M.C.M., Yeoh, E.K.: Measuring and preventing potentially avoidable hospital readmissions: a review of the literature. Hong Kong medical journal = Xianggang yi xue za zhi 16(5), 383-389 (2010)

59. Clemson, L., Mackenzie, L., Ballinger, C., Close, J.C.T., Cumming, R.G.: Environmental interventions to prevent falls in community-dwelling older people: a meta-analysis of randomized trials. Journal of aging and health 20(8), 954-971 (2008). doi: 10.1177/0898264308324672

60. Jette, A.M., Haley, S.M., Coster, W.J., Kooyoomjian, J.T., Levenson, S., Heeren, T., Ashba, J.: Late Life Function and Disability Instrument: I. Development and Evaluation of the Disability Component. Journal of Gerontology: Medical Sciences 57a(4), M209-M216 (2002)

61. Collin, C., Wade, D.T., Davies, S., Horne, V.: The Barthel ADL Index: A reliability study. International Disability Studies 10(2), 61-63 (1988). doi: $10.3109 / 09638288809164103$

62. Klein, R.M., Bell, B.: Self-care skills: behavioral measurement with Klein-Bell ADL scale. Archives of Physical Medicine and Rehabilitation 63(7), 335-338 (1982)

63. The EuroQol Group: EuroQol - a new facility for the measurement of health-related quality of life. Health Policy 16(3), 199-208 (1990). doi: 10.1016/01688510(90)90421-9

64. Graham, J.E., Granger, C.V., Karmarkar, A.M., Deutsch, A., Niewczyk, P., DiVita, M.A., Ottenbacher, K.J.: The Uniform Data System for Medical Rehabilitation: Report of Follow-up Information on Patients Discharged from Inpatient Rehabilitation Programs in $2002-2010$. American journal of physical medicine \& rehabilitation / Association of Academic Physiatrists 93(3), 231-244 (2014). doi: 10.1097/PHM.0b013e3182a92c58

65. Tooth, L.R., McKenna, K.T., Smith, M., O'Rourke, P.K.: Reliability of scores between stroke patients and significant others on the Reintegration to Normal Living (RNL) Index. Disability and rehabilitation 25(9), 433-440 (2003). doi: 10.1080/0963828031000069726

66. Linacre, J.M., Heinemann, A.W., Wright, B.D., Granger, C.V., Mamilton, B.B.: The Structure and Stability of the Functional Independence Measure. Archives of Physical Medicine and Rehabilitation(75) (1994)

67. Barberger-Gateau, P., Dartigues, J.F., Letenneur, L.: Four Instrumental Activities of Daily Living Score as a predictor of one-year incident dementia. Age and ageing 22(6), 457-463 (1993). doi: 10.1093/ageing/22.6.457

68. Katz, S., Downs, T.D., Cash, H.R., Grotz, R.C.: Progress in development of the index of ADL. The Gerontologist 10(1), 20-30 (1970). doi: 10.1093/geront/10.1_part_1.20

69. van Swieten, J.C., Koudstaal, P.J., Visser, M.C., Schouten, H.J., van Gijn, J.: Interobserver agreement for the assessment of handicap in stroke patients. Stroke 19(5), 604-607 (1988). doi: 10.1161/01.str.19.5.604

\section{Figures}

Page $18 / 20$ 


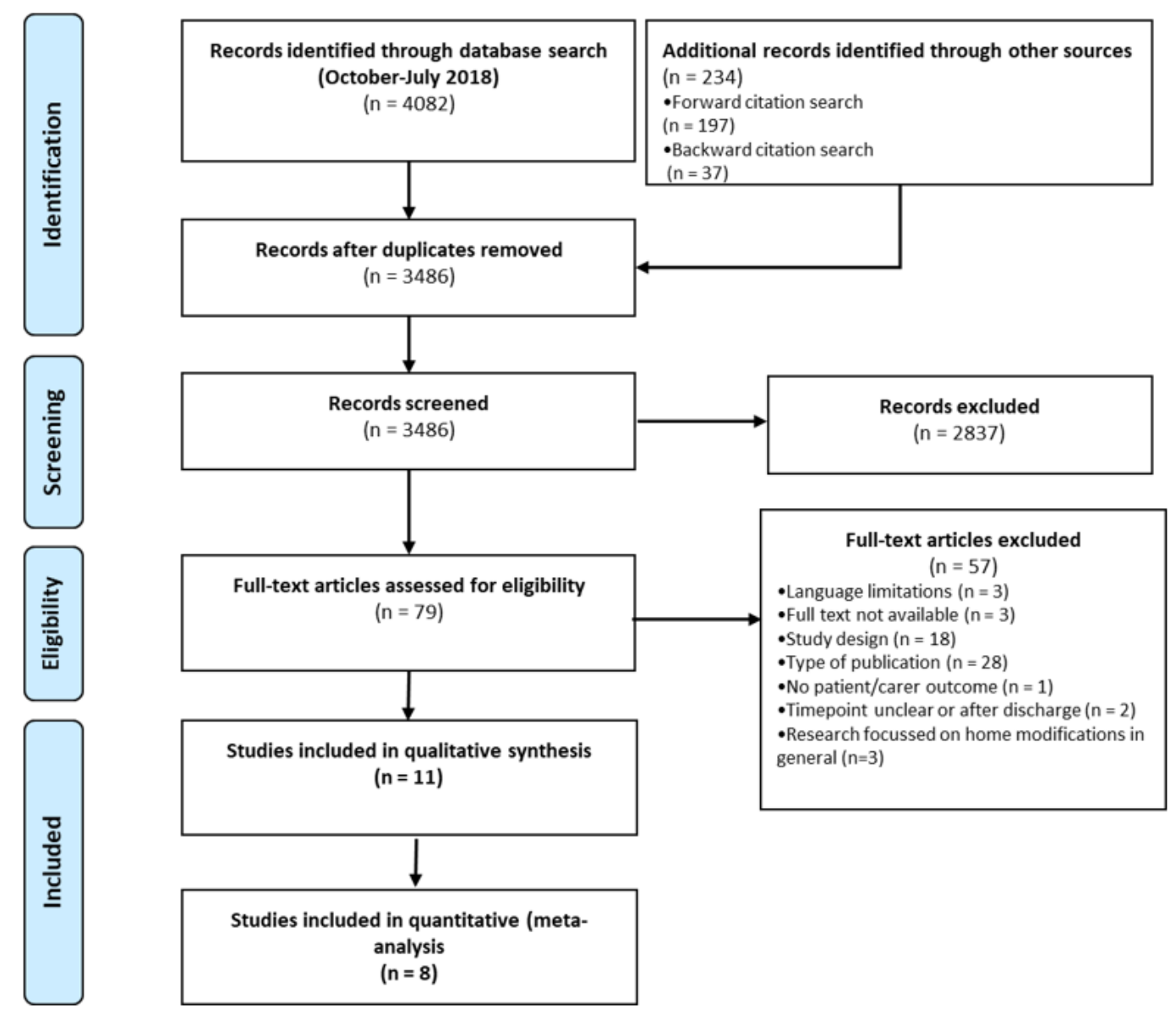

Figure 1

Flow diagram of study selection 


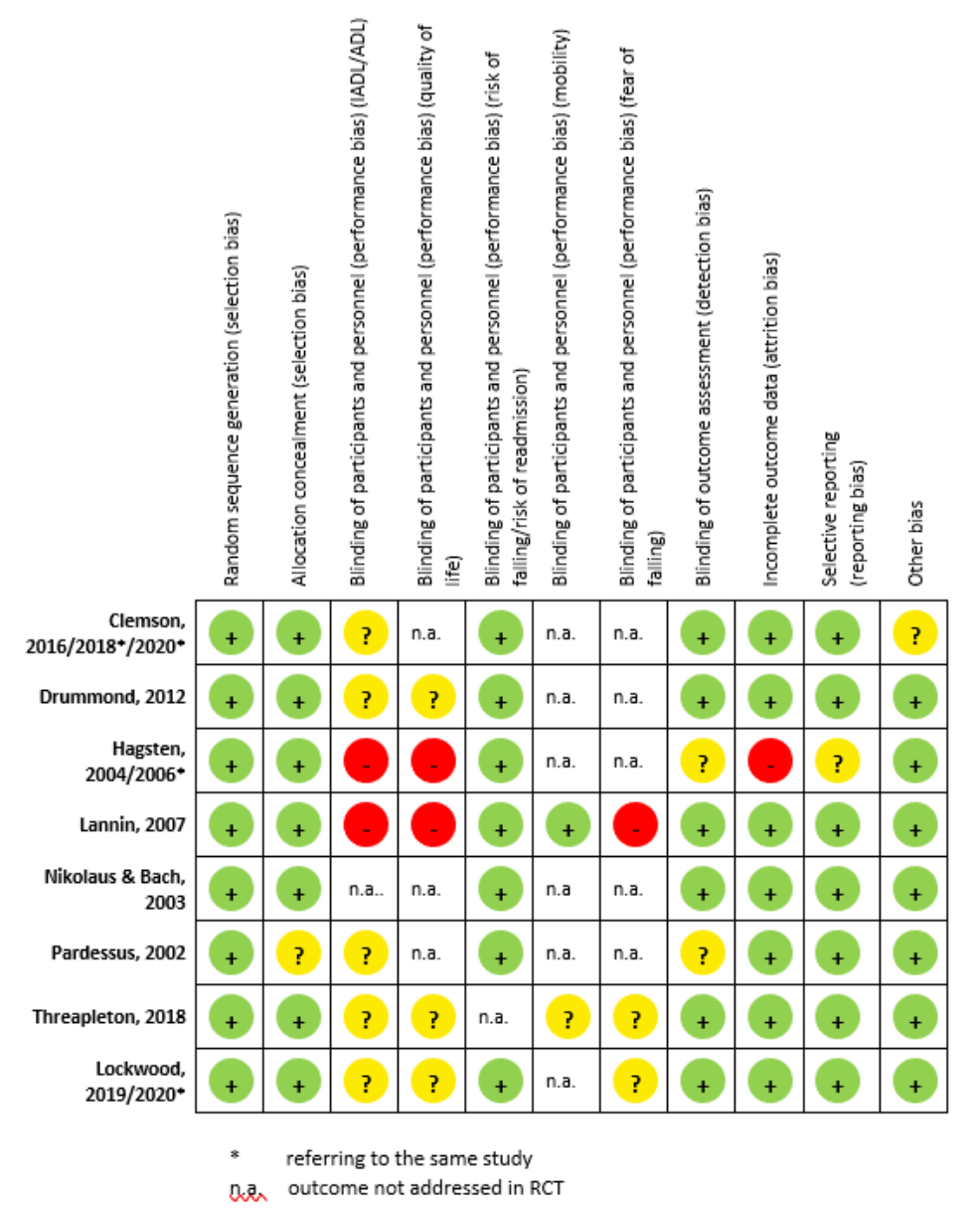

Figure 2

Risk of bias in single studies

\section{Supplementary Files}

This is a list of supplementary files associated with this preprint. Click to download.

- AdditionalFile1 searchstrategyupdate.docx

- AdditionalFile2Outcomemeasuresupdate.docx

- Additionalfile3DetailedROBsinglestudiesupdate.docx

- AdditionalFile4Qualityappraisalqualstudiesupdate.docx

- AdditionalFile5GRADEpooledeffectsupdate.docx

- Additionalfile6Forestplotsupdate.docx

- Additionalfile7Descriptivethemesupdate.docx

- Additionalfile8Analyticalthemesupdate.docx

- AdditionalFile9Integrativesynthesis.docx

- RevisedPRISMAchecklistPredischargemanuscript.doc 